\title{
Deletion of either the regulatory gene ara 1 or metabolic gene $x k i 1$ in Trichoderma reesei leads to increased CAZyme gene expression on crude plant biomass
}

Tiziano Benocci ${ }^{1}$, Maria Victoria Aguilar-Pontes ${ }^{1}$, Roland Sándor Kun ${ }^{1}$, Ronnie J. M. Lubbers', Kathleen Lail ${ }^{2}$, Mei Wang ${ }^{2}$, Anna Lipzen², Vivian Ng${ }^{2}$, Igor V. Grigoriev ${ }^{2,3}$, Bernhard Seiboth ${ }^{4}$, Paul Daly ${ }^{1}$ and Ronald P. de Vries ${ }^{1 *}$ (])

\begin{abstract}
Background: Trichoderma reesei is one of the major producers of enzymes for the conversion of plant biomass to sustainable fuels and chemicals. Crude plant biomass can induce the production of CAZymes in T. reesei, but there is limited understanding of how the transcriptional response to crude plant biomass is regulated. In addition, it is unknown whether induction on untreated recalcitrant crude plant biomass (with a large diversity of inducers) can be sustained for longer. We investigated the transcriptomic response of T. reesei to the two industrial feedstocks, corn stover (CS) and soybean hulls (SBH), over time ( $4 \mathrm{~h}, 24 \mathrm{~h}$ and $48 \mathrm{~h}$ ), and its regulatory basis using transcription factor deletion mutants ( $\triangle x y r 1$ and $\triangle a r a 1)$. We also investigated whether deletion of a xylulokinase gene ( $\triangle x k i 1)$ from the pentose catabolic pathway that converts potential inducers could lead to increased CAZyme gene expression.

Results: By analyzing the transcriptomic responses using clustering as well as differential and cumulative expression of plant biomass degrading CAZymes, we found that corn stover induced a broader range and higher expression of CAZymes in T. reesei, while SBH induced more pectinolytic and mannanolytic transcripts. XYR1 was the major TF regulating CS utilization, likely due to the significant amount of D-xylose in this substrate. In contrast, ARA1 had a stronger effect on SBH utilization, which correlates with a higher abundance of L-arabinose in SBH that activates ARA1. Blocking pentose catabolism by deletion of $x k i 1$ led to higher expression of CAZyme encoding genes on both substrates at later time points. Surprisingly, this was also observed for $\triangle a r a 1$ at later time points. Many of these genes were XYR1 regulated, suggesting that inducers for this regulator accumulated over time on both substrates.
\end{abstract}

Conclusion: Our data demonstrates the complexity of the regulatory system related to plant biomass degradation in T. reesei and the effect the feedstock composition has on this. Furthermore, this dataset provides leads to improve the efficiency of a T. reesei enzyme cocktail, such as by the choice of substrate or by deleting $x k i 1$ to obtain higher production of plant biomass degrading CAZymes.

Keywords: Plant biomass degradation, Trichoderma reesei, Xylan, Cellulose, CAZymes, Xyr1

\footnotetext{
*Correspondence: r.devries@westerdijkinstitute.nl

${ }^{1}$ Fungal Physiology, Westerdijk Fungal Biodiversity Institute \& Fungal

Molecular Physiology, Utrecht University, Uppsalalaan 8, 3584 CT Utrecht,

The Netherlands

Full list of author information is available at the end of the article
} 


\section{Background}

Plant biomass is the most abundant renewable carbon source on Earth for conversion into biofuel and biochemicals by the biotechnology industry. It is composed of three major polysaccharides (cellulose, hemicellulose and pectin), and many different plant biomass degrading (PBD) enzymes are required for efficient degradation [1]. Trichoderma reesei (Hypocrea jecorina) is a cellulolytic filamentous saprobic fungus that has been used in biotechnology for many decades, mainly for cellulase production [2]. Major developments for industrial applications were reviewed previously [3], such as the improved-cellulase producer QM9414 strain or the hypersecreting RUT C30 strain (CCR derepressed, due to a partial truncation of CRE1) [4]. The disaccharide lactose is the primary industrial inducer of $T$. reesei cellulase production, but induction by crude plant biomass is a promising alternative. However, a challenge remains to sustain induction when the limited inducers that are available have been consumed. Sustaining induction for longer time periods will lead to higher overall CAZyme yields as well as a more diverse array of CAZymes and reduce enzyme production costs.

While $T$. reesei has been suggested as a model to study plant biomass degradation [5], its strategy to degrade plant biomass differs from most other saprobes studied so far, as its genome encodes a narrow set of enzymes, some of which (e.g., several cellulases) are produced at high levels [6]. Its molecular mechanisms for the production of plant biomass degrading enzymes and sugar catabolism have been studied [7-10], demonstrating that XYR1 is the major cellulolytic and xylanolytic regulator [8], but also involved in $\mathrm{D}$-xylose and (partially) L-arabinose catabolism. A second regulator, ARA1, regulates $\mathrm{L}$-arabinose and D-galactose releasing as well as catabolic enzymes in response to L-arabinose and D-galactose [9].

Carbon catabolism related to plant biomass conversion has been studied in many fungi and recently reviewed [11]. Carbon catabolism in T. reesei is peculiar, as, more enzymes play a role in multiple catabolic pathways, compared to several other saprobes [11]. For example, the $T$. reesei pentose catabolic pathway $(\mathrm{PCP})$ and the $\mathrm{D}$-galactose oxido-reductive pathway share three enzymes (Fig. 1), and each of these pathways are regulated by both XYR1 and ARA1 [9]. When genes of carbon catabolic pathways are deleted, this can lead to the accumulation of metabolites, which can function as inducers. In Aspergillus niger, a xylulokinase mutant (where the last step of the PCP is blocked), resulted in accumulation of inducers, such as xylitol and L-arabitol [12]. In T. reesei, xylitol and L-arabitol accumulation was observed in deletion strains $(\Delta x y l 1, \Delta l a d 1$ and $\Delta l x r 3)$ of three earlier steps of the PCP [13]. While there are no reports of the effect of a full deletion of the final step of the PCP, the xylulokinase (XKI1), its antisense inhibition increased xylitol production in T. reesei [14].

Transcriptomics is a sensitive tool to probe complex regulatory events, but only a few $T$. reesei transcriptomics studies have been performed using crude plant biomass [15-17], while others used polymers or mono- and disaccharides [3]. In particular, only one transcriptomic study analyzed a regulatory mutant $(\Delta x y r 1)$ using crude plant biomass (wheat bran) [18], whereas no catabolic mutants have been analyzed by transcriptomics using crude plant biomass. This previous study, analyzing only a single time point, identified a set of genes regulated by XYR1 (including not only (hemi-)cellulolytic genes, but also genes encoding non-enzymatic cellulose active enzymes, sugar transporters and heat shock proteins) [18]. Analysis across multiple time points is required to uncover the dynamic changes in gene expression patterns as the crude plant biomass is degraded by the fungus.

In this study, we analyzed the transcriptome of $T$. reese $i$ during growth on the two industrial substrates soybean hulls $(\mathrm{SBH})$ and corn stover (CS) over time, using two regulatory mutants $(\Delta x y r 1$ and $\Delta$ ara 1$)$ and one metabolic mutant ( $\Delta x k i 1)$. These two substrates have different polysaccharide compositions $[19,20]$, allowing us to deeply explore how the regulatory system responds to a wide array of sugar inducers released from these polysaccharides. CS is richer in hemicellulose, particularly arabinoxylan, while SBH is richer in pectin, xyloglucan and mannan (Additional file 1). This study resulted in three main findings. T. reesei had higher and broader transcript levels of PBD CAZyme genes when cultured on CS. ARA1 had a larger role in the regulation of PBD transcripts on SBH compared to XYR1, which was the major TF regulating plant biomass degradation in CS. The block of pentose catabolism (by deletion of $x$ ki1 from the $\mathrm{PCP}$ ) led to higher PBD CAZyme expression at later time points in the CS and $\mathrm{SBH}$ cultures.

\section{Results}

The regulatory and catabolic mutants had severely reduced growth on pure mono- and polysaccharides compared to the reference strain, but not on crude plant biomass

Three $T$. reesei deletion mutants $(\Delta x y r 1, \Delta a r a 1$ and $\Delta x k i 1)$ were phenotypically compared to the reference strain QM9414 by growth on various carbon sources, including mono- and disaccharides, polymers and crude plant biomass (Fig. 2). As reported previously [21, 22], deletion of xyr1 most severely affected growth on D-xylose, less severely on lactose and to an even lesser extent on L-arabinose, xylitol, arabinan 


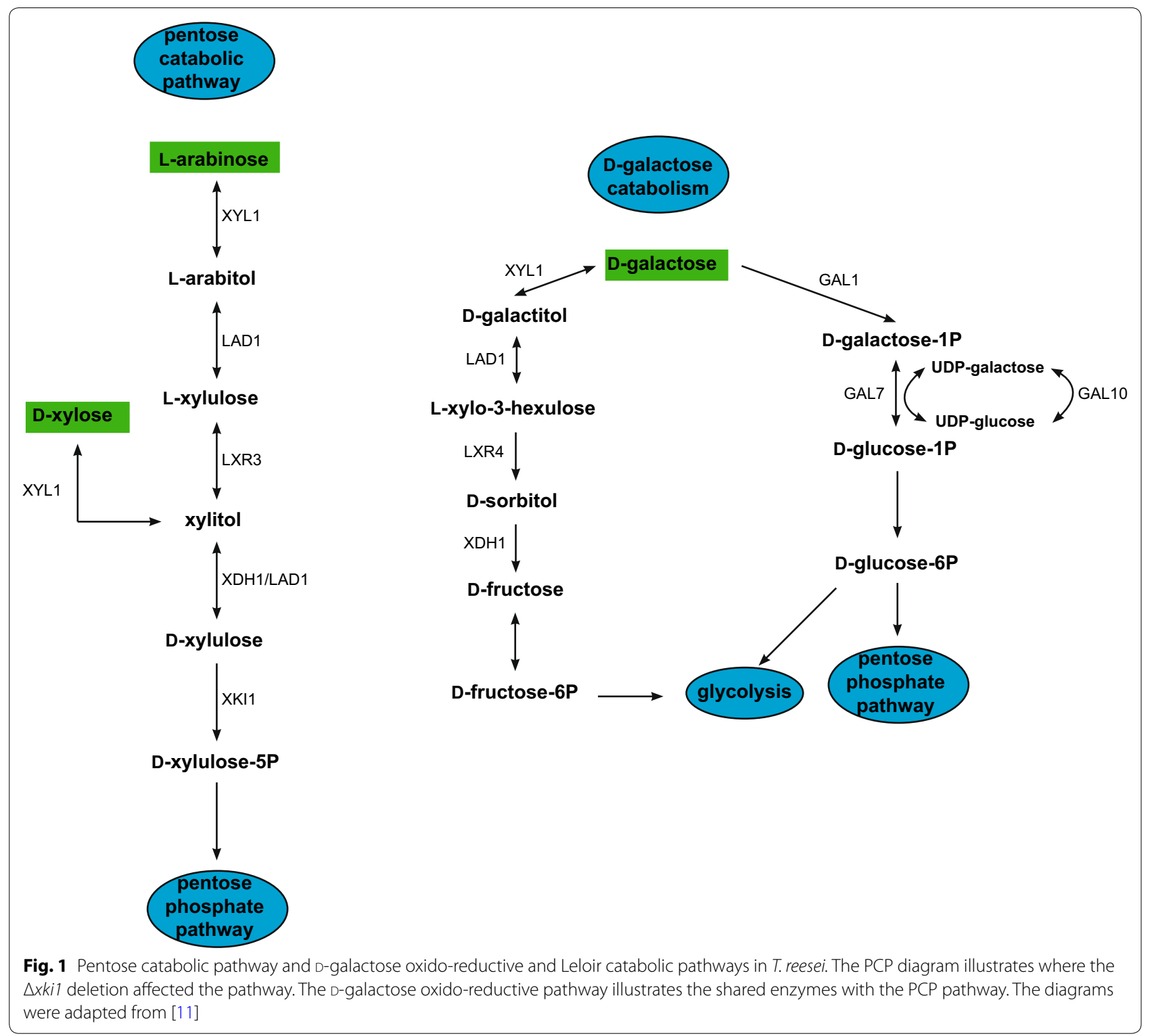

and arabinoxylan (Fig. 2). Deletion of ara1 abolished growth on D-galactose and reduced growth on L-arabinose, L-arabitol, while growth on D-xylose, arabinan, arabinoxylan and lactose was not affected (Fig. 2). Deletion of the xylulokinase xki1 resulted in strongly reduced growth on D-xylose and to a lesser extent on $\mathrm{L}$-arabinose and xylitol, while growth on L-arabitol was abolished (Fig. 2). In all three deletion strains, strong growth reduction was only observed on specific monoand polysaccharides, but not on crude plant biomass (Fig. 2), perhaps reflecting the diversity of nutrients available in plant biomass and highlighting the complex regulatory network during growth on crude substrates.
The $T$. reesei reference strain expressed PBD CAZymes at higher levels on corn stover than on soybean hulls $\mathrm{CS}$ or SBH cultures from transfer experiment were sampled after 4, 24 and $48 \mathrm{~h}$ for transcriptome analysis that focused on genes encoding PBD CAZymes, carbon catabolic enzymes and related TFs (Additional file 4). To investigate the adaptation to each substrate, we initially compared the PBD CAZyme transcriptome profiles by clustering all six conditions of the reference strain (Fig. 3). The $4 \mathrm{~h}$ and $24 \mathrm{~h} \mathrm{CS}$ samples clearly separated from the other samples, while the $4 \mathrm{~h}$ SBH sample was also distant from the remaining samples (Fig. 3). Interestingly, the $24 \mathrm{~h}$ and $48 \mathrm{~h} \mathrm{SBH}$ samples clustered with the $48 \mathrm{~h}$ CS sample (Fig. 3), and overall had less highly 


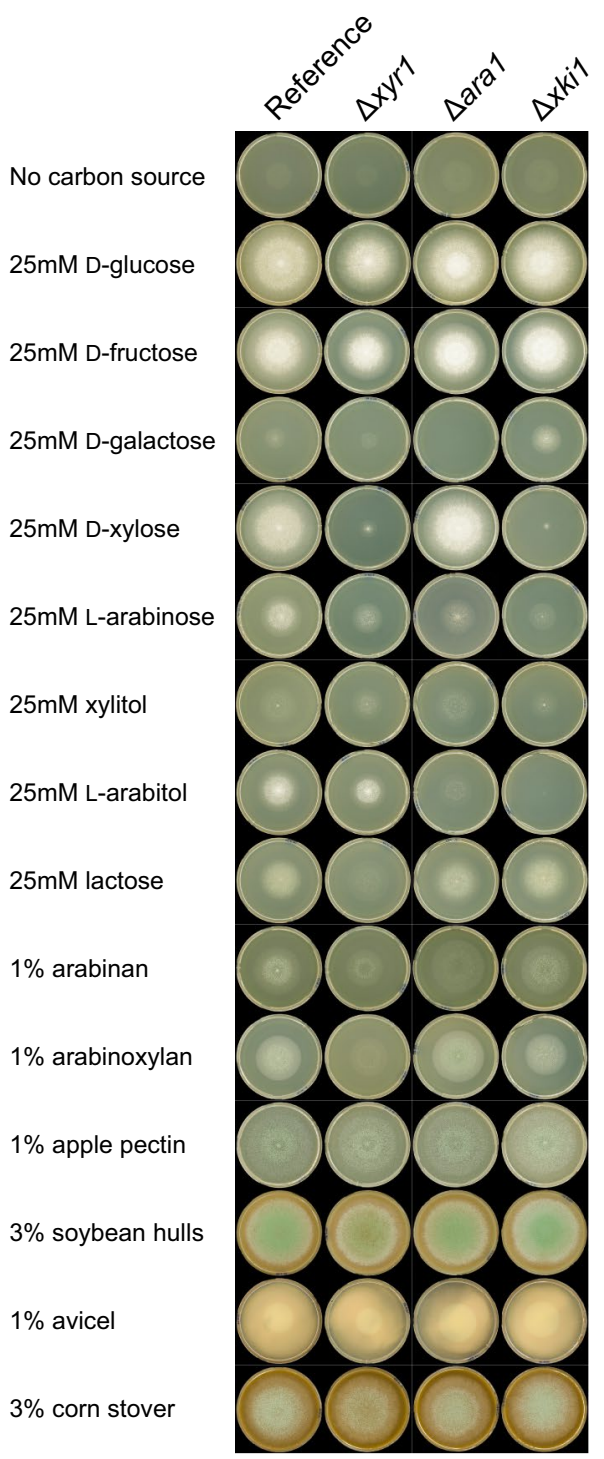

Fig. 2 Growth profile of T. reesei reference strain (QM9414) and deletion mutants $\triangle x y r 1, \triangle a r a 1$ and $\triangle x k i 1$ on mono- and disaccharides, polymers and crude plant biomass. All plates were incubated for 5 days at $28^{\circ} \mathrm{C}$. The growth on Avicel of all mutants was comparable with the reference strain

expressed genes than the other samples. This suggests that during growth on $\mathrm{SBH}$ the inducing compounds were more quickly removed from the cultures than on CS.

Deep transcriptomic analysis (Fig. 4), in terms of which PBDs were induced and the level of total expression, helped to elucidate the patterns found in the PBD CAZymes cluster analysis (Fig. 3). CS induced more as well as higher total expression of cellulolytic and xylanolytic genes (Fig. 4a, b). In contrast, SBH induced more as well as higher total expression of mannanolytic, amylolytic and pectinolytic genes at the initial stage (4 h) (Fig. 4). This indicates that $T$. reesei is able to sense the major inducers from each substrate, adapting the expression of PBD CAZyme encoding genes to the substrate composition.

CS induced more PBD CAZyme encoding genes compared to SBH (Fig. 3) and to a higher level of total expression at all three time points (Fig. 4). The peak of PBD CAZyme gene expression on CS was at $4 \mathrm{~h}$ where the total expression of xylanolytic genes was highest (such as $x y n 1 / 2 / 3 / 4$, bxl1, aes1, agu1 and abf2) (Fig. 4b, Additional file 4), while cellulolytic gene expression was highest at $24 \mathrm{~h}$ (e.g., cbh1/2, egl1/2/3//5 and two LPMOs egl4 and cel61b) (Fig. 4b, Additional file 4). In general, in both substrates, the number of induced PBD CAZyme encoding genes and the total expression level decreased over time (Fig. 4), with a steeper decline over time in the total expression on SBH compared to CS (Fig. 4). The decrease over time led to a total level of PBD CAZyme gene expression at $48 \mathrm{~h}$ on both substrates that is comparable to the level in the D-fructose pre-cultures (Figs. 3, 4b) indicating that at $48 \mathrm{~h}$ little or no inducers were present in the cultures from both substrates. Considering that studies in A. niger [23, 24] and $T$. reesei [25] indicated that inducer concentrations below $1 \mathrm{mM}$ already activate the regulatory systems, this suggests that in fact no inducers are present anymore.

Total expression of carbon catabolic genes in CS and SBH followed the PBD cellulolytic gene expression matches with CAZyme patterns where the expression decreased over time in both substrates, but for this gene group the total expression was comparable in both substrates at the same time point (Fig. 5). Similar to what was observed for the CAZyme genes, a more clear adaptation to substrate composition was observed at $4 \mathrm{~h}$ than at the later time points (Fig. 5). The total expression of genes involved in both the PCP and D-galactose oxidoreductive pathway ( $x y l 1, \operatorname{lad} 1$ and $x d h 1)$ was higher in CS, while in SBH the total expression of the L-rhamnose pathway was higher (Fig. 5b). Transcriptional regulator encoding genes appeared to be similarly induced in both substrates (Fig. 6), but small differences were observed that also correlated with the composition of the substrates. CS induced more highly xyr1 and ace 3 (both (hemi-)cellulolytic activators [26, 27]), while SBH higher expressed rhr1 (ortholog of the A. niger rhamnose responsive regulator rhaR [28]) after $4 \mathrm{~h}$, as well as xpp1 (xylanase repressor [29]). The two substrates demonstrated clear differences in their induction pattern and physiological response and this led us to initially analyze the effects of the three deletion mutants by each substrate separately and then compare these analyses. 

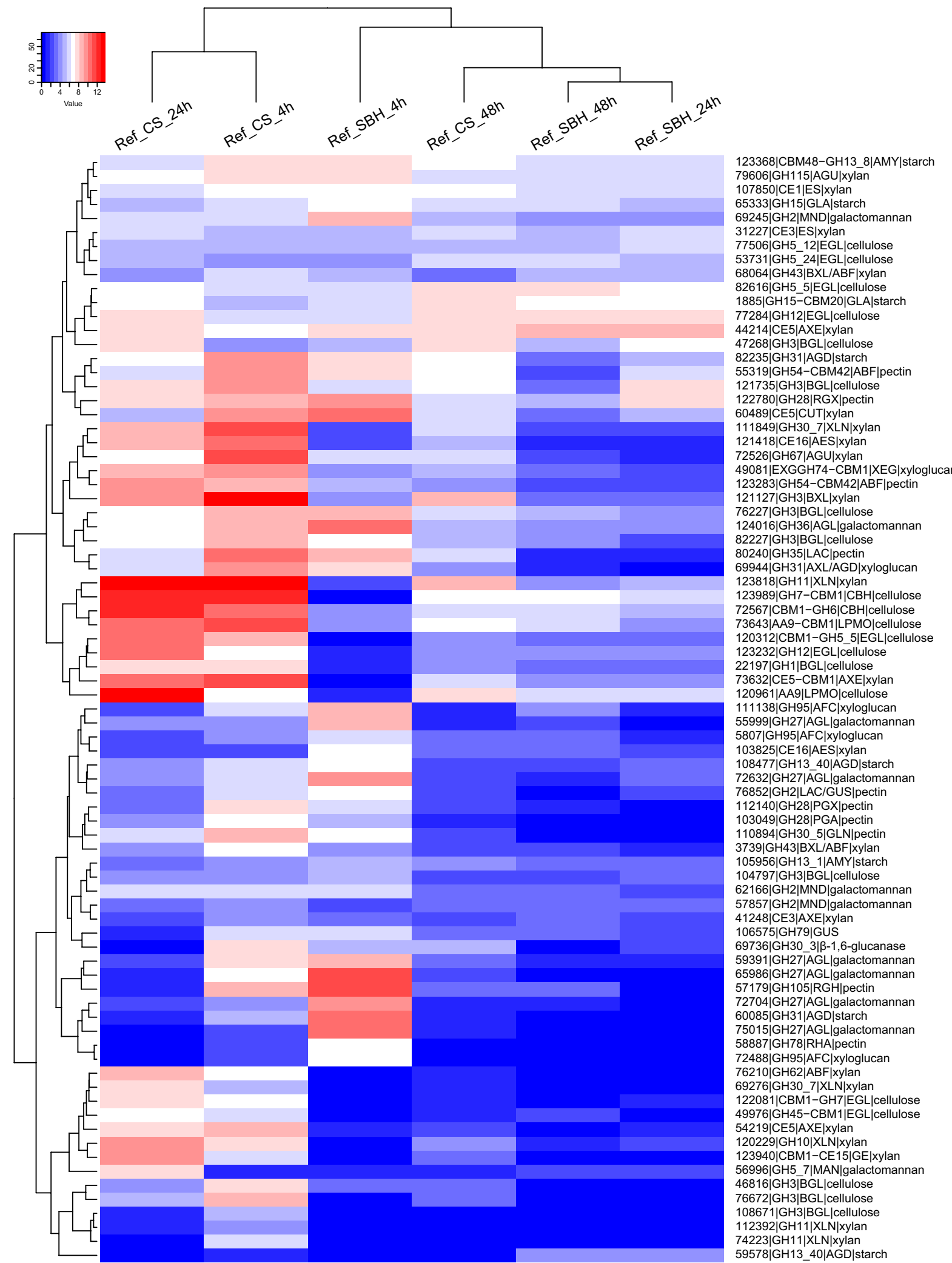

Fig. 3 Hierarchical clustering (Euclidean distance) of PBD CAZyme gene expression in the T. reesei reference strain on corn stover (CS) and soybean hulls (SBH) 


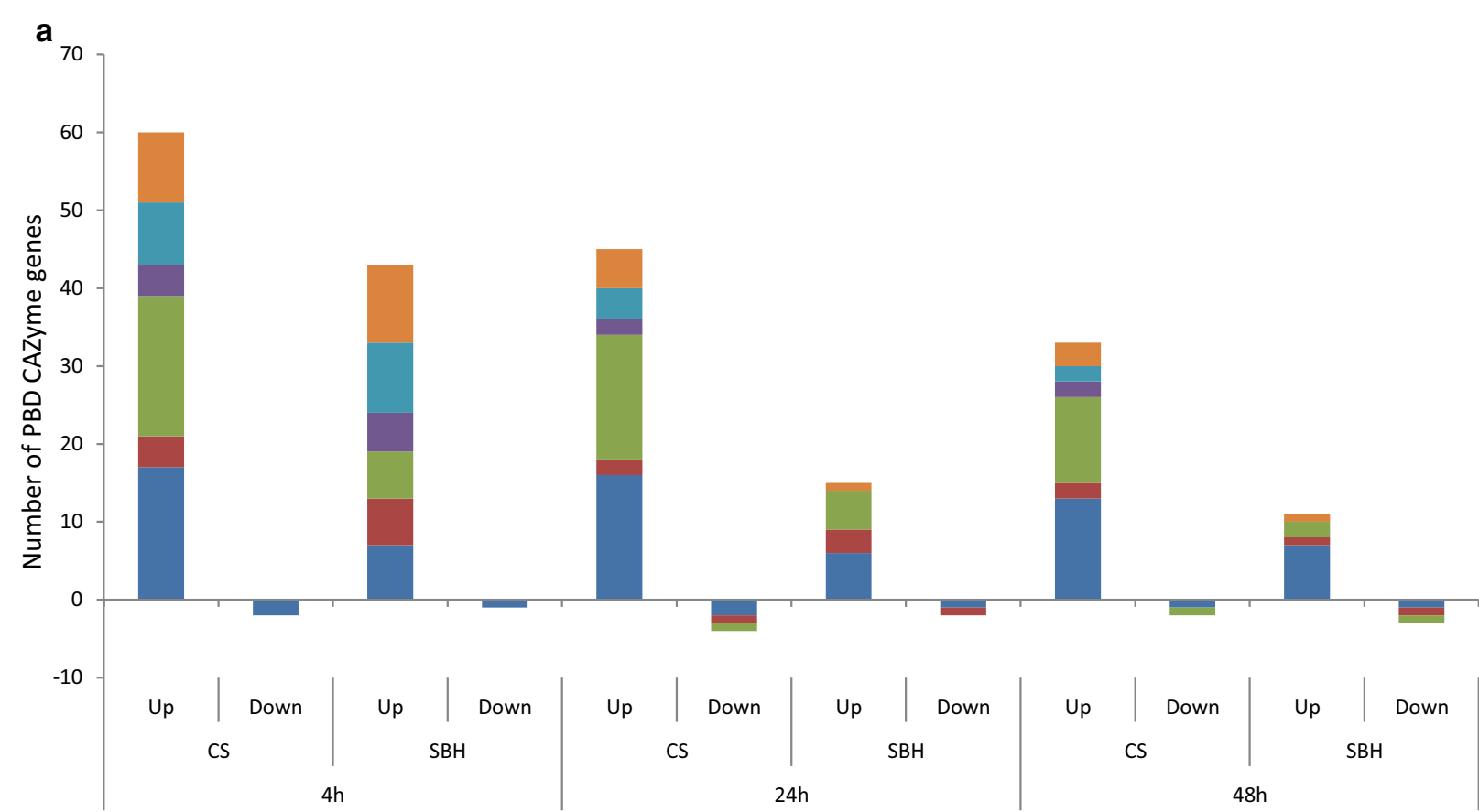

b

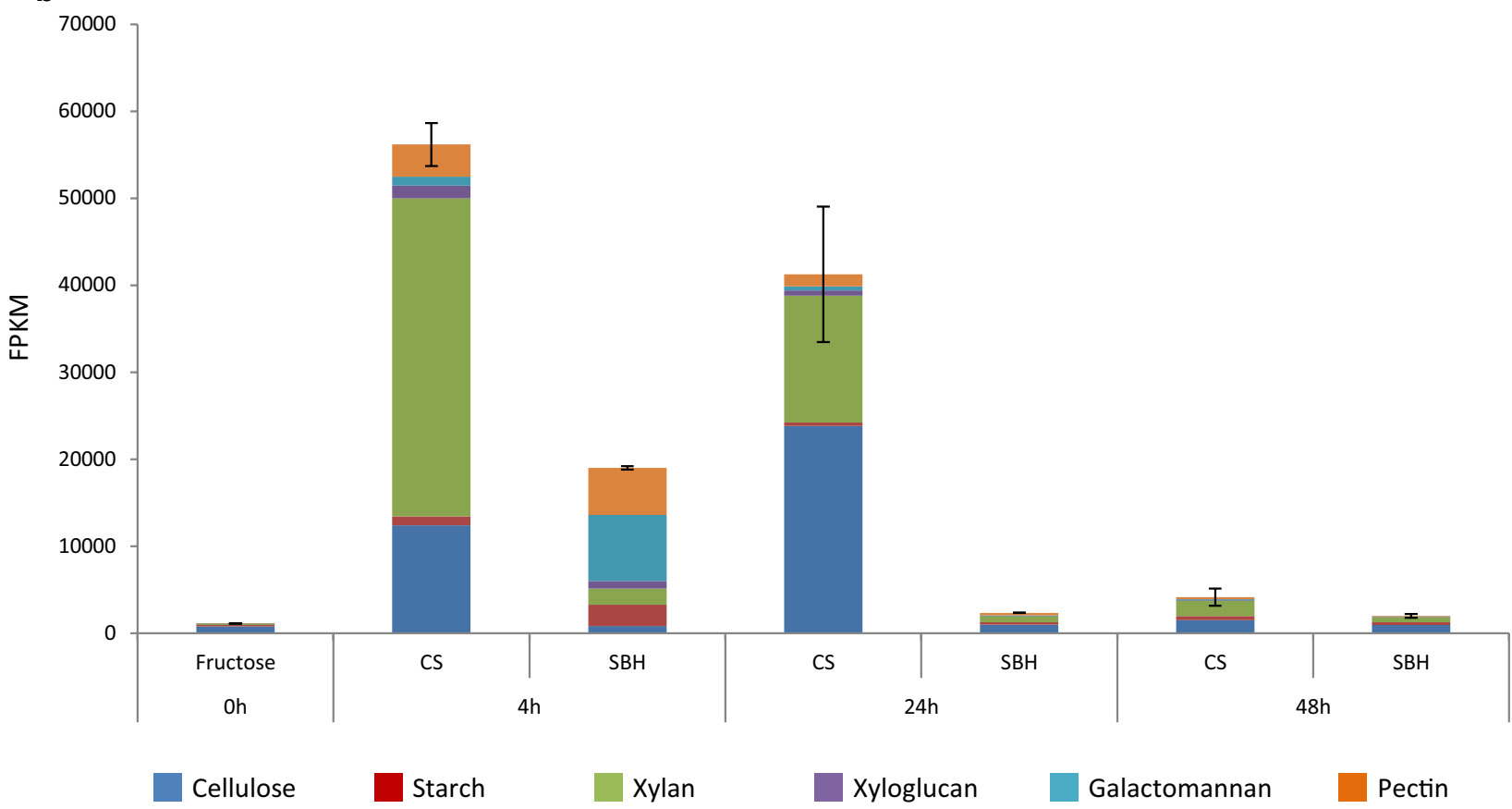

Fig. 4 Comparison of PBD CAZyme gene expression in the reference strain (QM9414) on corn stover (CS) and soybean hulls (SBH). a Fold change analysis. Differentially expressed genes were those with a $p$ value $\leq 0.05$, fold change $>2.5$ (log2foldchange $>1.32$ ) compared to pre-culture and FPKM $\geq 18$ in at least one condition. b Total expression analysis were performed with average FPKM level between three replicates in plant biomass and two replicates in pre-culture. Error bars represent the standard error on the total PBD CAZyme expression 

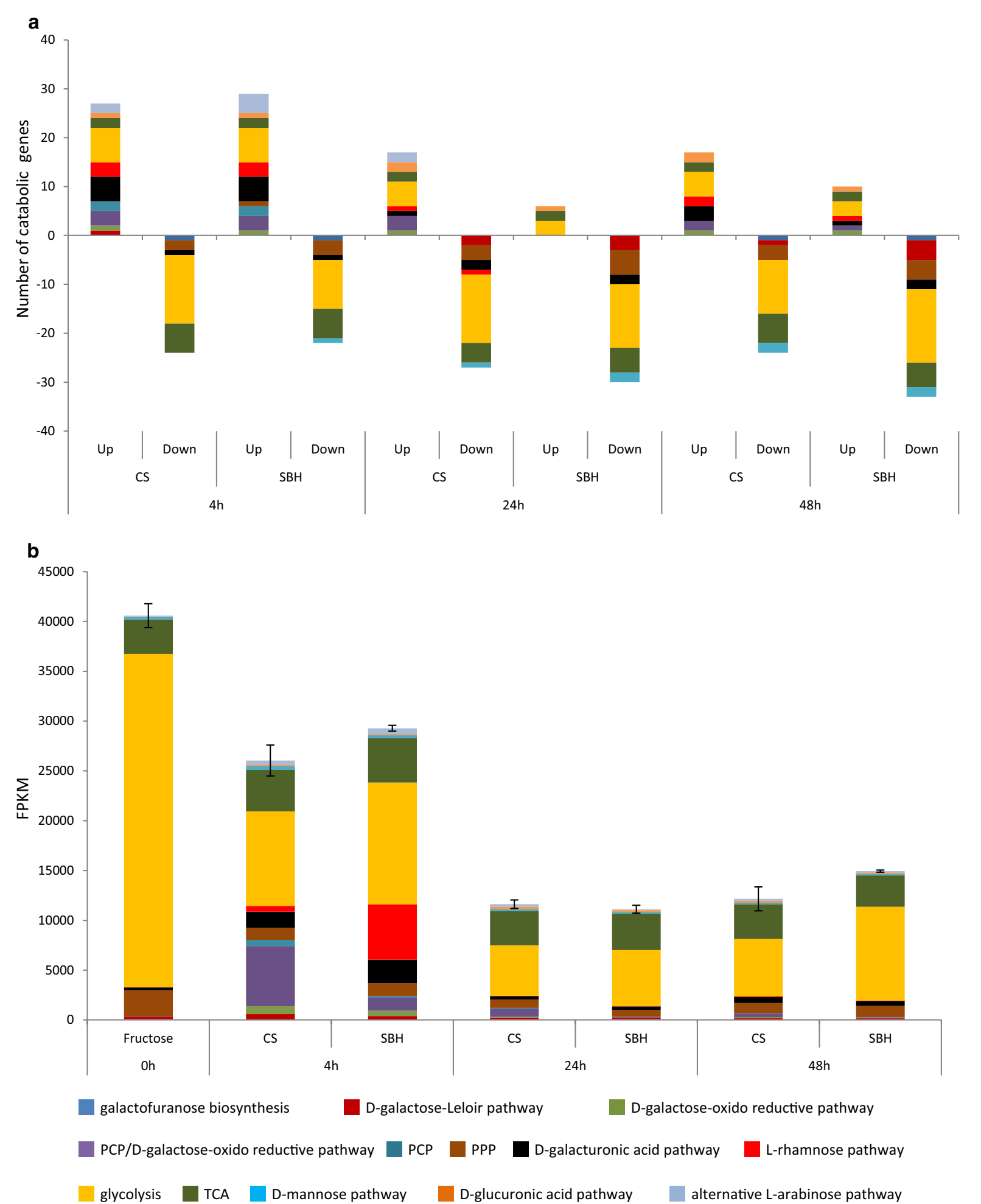

Fig. 5 Comparison of reference carbon catabolic gene expression in corn stover and soybean hulls. a Fold change analysis. Differentially expressed genes were those with a $p$ value $\leq 0.05$, fold change $>2.5$ (log2foldchange $>1.32$ ) compared to pre-culture and FPKM $\geq 18$ in at least one condition. b Total expression analysis were performed with average FPKM level between 3 replicates in plant biomass and 2 replicates in pre-culture. Error bars represent the standard error on the total C-catabolism expression 


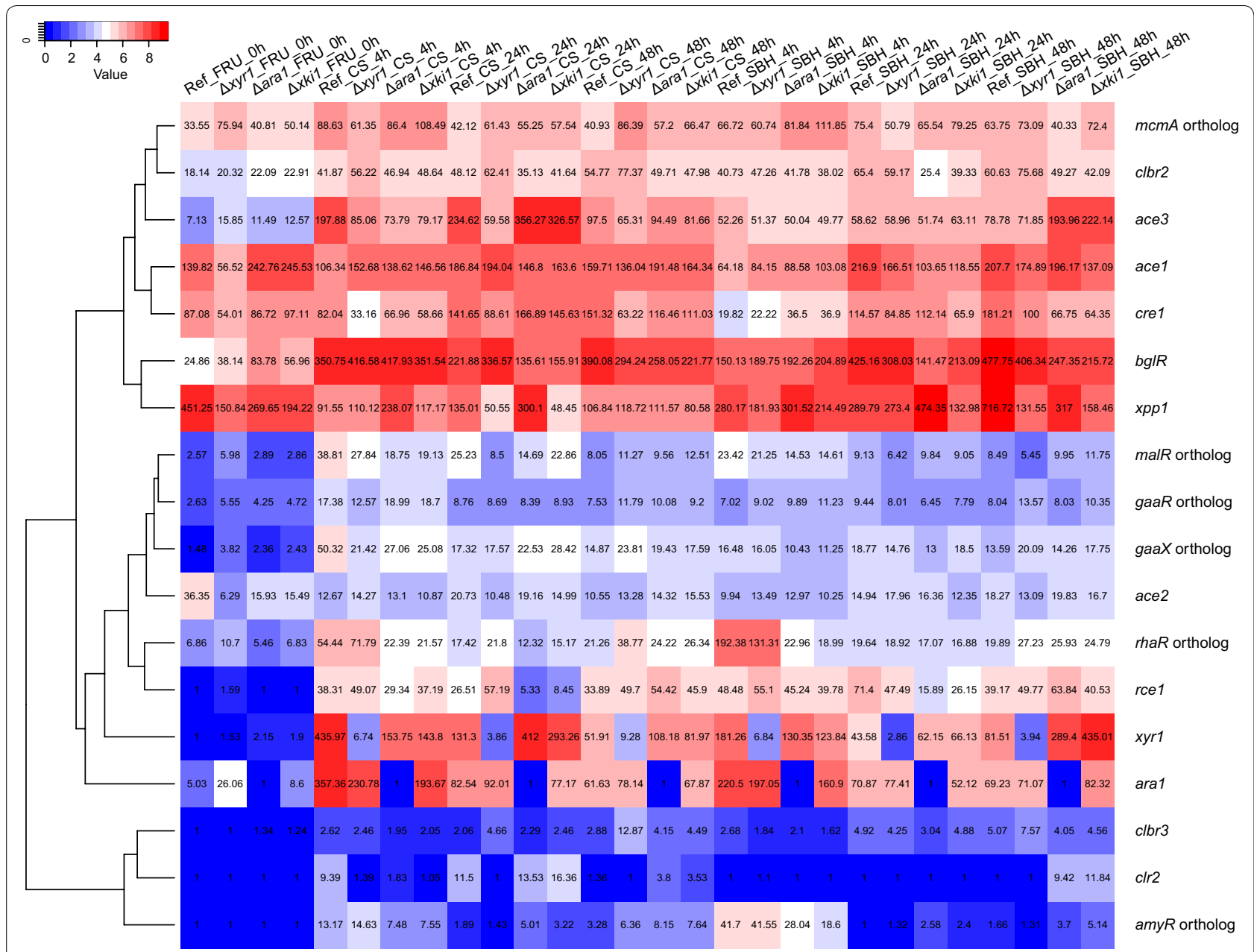

Fig. 6 Hierarchical clustering (Euclidean distance) of regulator gene expression in all strains in D-fructose (pre-culture), corn stover and soybean hulls

\section{XYR1 is the major TF regulating the degradation of corn stover, while ARA1 more strongly affects soybean hulls utilization}

We initially analyzed the secretome profiles of the reference strain and what we considered phenotypically the most extreme mutant, $\Delta x y r 1$, by SDS-PAGE gel analysis on later time points ( $24 \mathrm{~h}$ and $48 \mathrm{~h}$ ), where we expected to see clear patterns. T. reesei reference strain secreted a different protein pattern in each substrate, where CS showed more intense bands especially around $25 \mathrm{kDa}$. According to our expectations, xyr1 deletion caused a severe secretome reduction in terms of bands intensity and range, in both substrates (Additional file 6).

Clustering analysis showed that in CS, the $4 \mathrm{~h}$ and $24 \mathrm{~h}$ samples of the reference, $\Delta a r a 1$ and $\Delta x k i 1$ cluster as two related, time-dependent clusters, indicating that overall these strains behave very similar during growth on CS (Fig. 7). At $48 \mathrm{~h}$, these strains still cluster together, but are now related to the $24 \mathrm{~h}$ and $48 \mathrm{~h}$ samples of $\Delta x y r 1$, characterized by an overall reduction in gene expression compared to the reference strain at the earlier time points (Fig. 7). The $4 \mathrm{~h}$ sample of $\Delta x y r l$ is clearly distinct from the other samples, with also an overall low expression of the CAZyme genes, but with some genes that are still higher expressed than at later time points in this strain. The results, therefore, support a central role for XYR1 in degradation of CS.

A very different pattern was observed on SBH. Initially, there appears to be only a minor effect of $x y r 1$ as at $4 \mathrm{~h}$, the $\Delta x y r 1$ sample clusters with the $4 \mathrm{~h}$ reference strain sample (Fig. 8). However, at later time points the $\Delta x y r 1$ samples are again characterized by overall very low gene expression compared to the reference strain at the earlier time point. The $4 \mathrm{~h}$ samples of $\Delta a r a 1$ and $\Delta x k i 1$ cluster together, but distant from the reference strain and $\Delta x y r 1$ at this time point. However, they have some similarity to the later time points of $\Delta x y r l$, characterized by a low expression of part of the CAZyme 

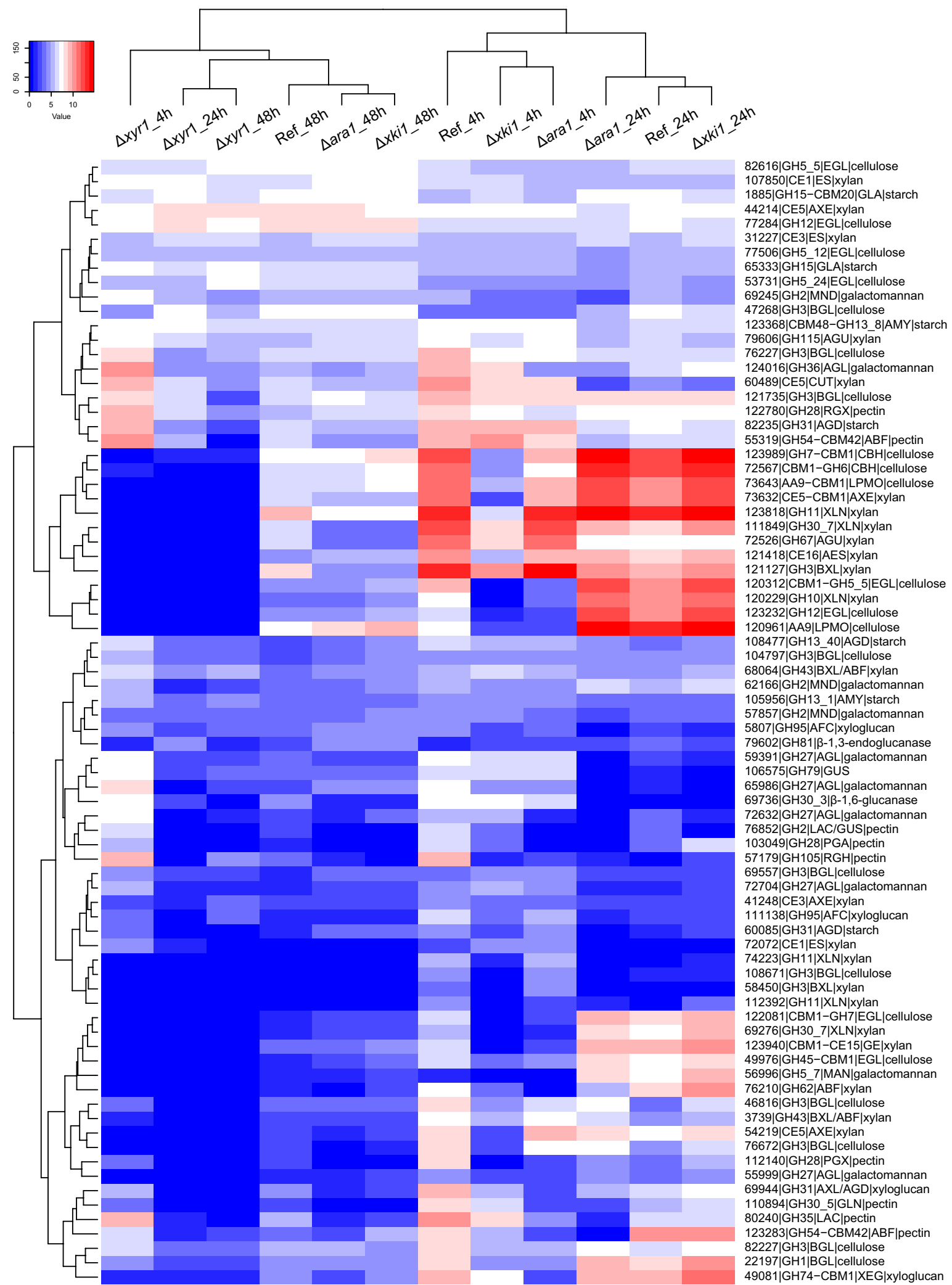

Fig. 7 Hierarchical clustering (Euclidean distance) of PBD CAZyme gene expression in the T. reesei reference strain (QM9414) and deletion mutants $\triangle x y r 1, \Delta a r a 1$ and $\triangle x k i 1$ on corn stover (CS) 

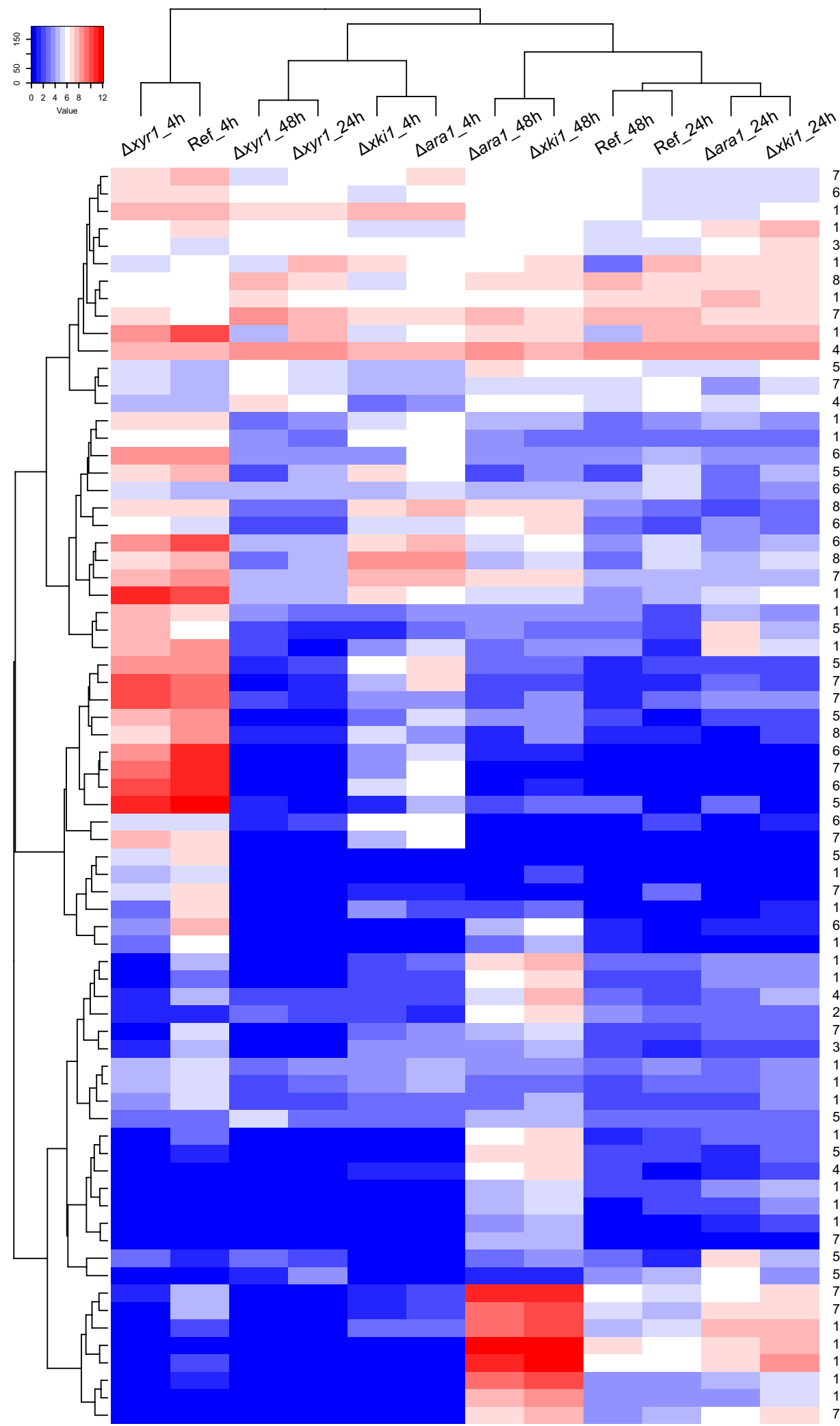

79606|GH115|AGU|xylan 65333|GH15|GLA|starch 23368|CBM48-GH13_8|AMY|starch 107850|CE1|ES|xylan 31227|CE3|ES|xylan 121735|GH3|BGL|cellulose 82616|GH5_5|EGL|cellulose 885|GH15-CBM20|GLA|starch 77284|GH12|EGL|cellulose 122780|GH28|RGX|pectin 44214|CE5|AXE|xylan 53731|GH5 24|EGL|cellulose 77506|GH5_12|EGL|cellulose 47268|GH3|BGL|cellulose 108477|GH13_40|AGD|starch 106575|GH79|GUS

69245|GH2|MND|galactomannan 55319|GH54-CBM42|ABF|pectin 68064|GH43|BXL/ABF|xylan 82227|GH3|BGL|cellulose 62166|GH2|MND|galactomannan 60489|CE5|CUT|xylan 82235|GH31|AGD|starch 76227|GH3|BGL|cellulose 124016|GH36|AGL|galactomannan 103825|CE16|AES|xylan 5807|GH95|AFC|xyloglucan 111138|GH95|AFC|xyloglucan 59391|GH27|AGL|galactomannan 72704|GH27|AGL|galactomannan 72632|GH27|AGL|galactomannan 55999|GH27|AGL|galactomannan 80240|GH35|LAC|pectin 60085|GH31|AGD|starch 75015|GH27|AGL|galactomannan 65986|GH27|AGL|galactomannan 57179|GH105|RGH|pectin 69736|GH30 3|ß-1,6-glucanase 72488|GH95|AFC|xyloglucan 58887|GH78|RHA|pectin 103049|GH28|PGA|pectin 76852|GH2|LAC/GUS|pectin 110894|GH30 5|GLN|pectin 69944|GH31|AXL/AGD|xyloglucan 112140|GH28|PGX|pectin $121127|\mathrm{GH} 3| \mathrm{BXL}$ |xylan 111849|GH30_7|XLN|xylan 49081|EXGGH74-CBM1|XEG|xyloglucan 22197|GH1|BGL|cellulose

72526|GH67|AGU|xylan 3739|GH43|BXL/ABF|xylan 105956|GH13_1|AMY|starch 104797|GH3|BGL|cellulose 123283|GH54-CBM42|ABF|pectin 57857|GH2|MND|galactomannan 121418|CE16|AES|xylan 56996|GH5_7|MAN|galactomannan 49976|GH45-CBM1|EGL|cellulose 120229|GH10|XLN|xylan 122081|CBM1-GH7|EGL|cellulose 123940|CBM1-CE15|GE|xylan 76672 |GH3|BGL|cellulose 58802|GH95|AFC|xyloglucan 59578|GH13 40|AGD|starch 72567|CBM1-GH6|CBH|cellulose 73643|AA9-CBM1|LPMO|cellulose 123818|GH11|XLN|xylan 123989|GH7-CBM1|CBH|cellulose 120961|AA9|LPMO|cellulose 123232|GH12|EGL|cellulose 120312|CBM1-GH5_5|EGL|cellulose 73632|CE5-CBM1|AXE|xylan

Fig. 8 Hierarchical clustering (Euclidean distance) of PBD CAZyme gene expression in the T. reesei reference strain (QM9414) and deletion mutants $\triangle x y r 1, \triangle a r a 1$ and $\triangle x k i 1$ on soybean hulls $(\mathrm{SBH})$ 
genes (Fig. 8). Some genes that are lowly expressed in the $\Delta a r a 1$ and $\Delta x k i 1$ strain at $4 \mathrm{~h}$ increase strongly in expression at the later time points, especially at $48 \mathrm{~h}$, but this is not $(\Delta x y r 1)$ or much less (reference strain) the case for the other strains. Overall, ARA1 appears to have a stronger role in SBH degradation, especially at the early time points. Interestingly, the metabolic mutant always clustered with $\Delta a r a 1$ at each time point on both substrates (Figs. 7, 8), which was unexpected, as the $\Delta x k i 1$ was expected to result in inducer accumulation and, therefore, upregulation of the ARA1 and XYR1 target genes, while $\Delta a r a 1$ was expected to reduce expression of a subset of those genes.

More detailed analysis confirmed that $\Delta x y r 1$ was the regulatory mutant more affected in CS, resulting in a lower total expression and number of PBD CAZymes in every time point (Fig. 9a, b). In contrast to CS, on SBH the $x y r 1$ deletion had a smaller effect on the response in all of the time points compared to the other mutants, in terms of number of PBD CAZymes induced and their total expression level (Fig. 9c, d). In SBH the biggest reduction in gene expression for $\triangle x y r 1$ was observed at $4 \mathrm{~h}$, but only approximately half of the PBD CAZyme genes were affected compared to CS and the total expression level was similar to the reference (Fig. 9). The ara1 deletion had a much smaller effect on gene expression on CS, confirming the higher importance of XYR1 in CS utilization (Fig. 9a, b).

The number of PBD CAZymes affected in both of regulatory mutants after $4 \mathrm{~h}$ on CS was similar but the total expression level in $\Delta a r a 1$ was still comparable to the reference (Fig. 9a, b). This was mainly due to xylanolytic genes, which remained expressed in $\Delta$ ara1, but were severely affected in $\Delta x y r 1$. At $4 \mathrm{~h}$ in CS, carbon catabolism was also affected by both mutations. The PCP genes were lowly expressed in both regulatory mutants, but the D-galactose Leloir and oxido-reductive pathways were affected more by the ara1 deletion, while L-rhamnose and D-galacturonic pathways were affected only by the ara 1 deletion (Fig. 10a, b).

Similar to CS, the initial response on SBH was also affected by both regulatory mutants, but the effect was larger for $\Delta a r a 1$ than for $\Delta x y r 1$ (Fig. 9c, d). In $\Delta a r a 1$ induction of mannanolytic and pectinolytic genes were particularly reduced (Fig. 9c, d). In addition, genes of the $\mathrm{PCP}$, both $\mathrm{D}$-galactose pathways and the $\mathrm{D}$-galacturonic acid pathway (only at $4 \mathrm{~h}$ ) were severely reduced in $\Delta$ ara 1, while the L-rhamnose pathway genes were not or lowly expressed in $\Delta$ ara1 (Fig. 10c, d). These pathways were not substantially affected in the xyr1 mutant on SBH. This indicates that $\Delta a r a 1$ was not/less able to catabolize or release $\mathrm{D}$-galactose, $\mathrm{L}$-arabinose and L-rhamnose, confirming that ARA1 has a larger effect on SBH utilization than XYR1.

Surprisingly, in contrast to $\Delta x y r 1$ and the initial response, at $24 \mathrm{~h}$ in CS and $24 \mathrm{~h}$ and $48 \mathrm{~h}$ in SBH $\Delta$ ara 1 induced more PBD CAZymes and to a higher level compared to the reference (Fig. 9). The same phenomenon was observed also for the $\Delta x k i 1$ strain and will be described in the following section.

\section{Deletion of $x k i 1$ or ara 1 leads to higher expression of cellulolytic and xylanolytic genes in soybean hulls and corn stover}

Clustering analysis (Figs. 7, 8) showed that $\Delta x k i 1$ and $\Delta$ ara1 samples always clustered together and by time point in both substrates, suggesting a similar response from each of the mutants, which was confirmed by transcriptomic analysis where PBD CAZyme genes (Fig. 9) and carbon catabolic genes (Fig. 10) were similarly expressed. In $\Delta x k i 1$ and $\Delta a r a 1$, the initial responses $(4 \mathrm{~h})$ to both substrates was severely affected, while at later time points ( $24 \mathrm{~h}$ in CS, $24 \mathrm{~h}$ and $48 \mathrm{~h}$ in SBH) more PBD CAZyme genes were induced and at higher level compared to the reference (Fig. 9). Remarkably, $\Delta x k i 1$ appeared to be more extreme in its responses to both substrates compared to $\Delta a r a 1$ (Fig. 9). The $\Delta x k i 1$ initial reduction in gene expression $(4 \mathrm{~h})$ to both substrates, but especially on CS, was more affected than that of $\triangle$ ara 1 , while during the PBD CAZyme gene expression peaks (24 $\mathrm{h}$ in CS, $24 \mathrm{~h}$ and $48 \mathrm{~h}$ in SBH) more PBD CAZyme genes were highly expressed compared to $\triangle$ ara1 (Fig. 9a, c). At these time points, and especially in $\mathrm{SBH}$, genes of the PCP, D-galactose pathways and D-galacturonic acid pathway were also higher expressed in both strains (Fig. 10b, d). Many of the genes that were upregulated in one or both of the strains ( $\Delta x k i 1$ and/or $\Delta a r a 1)$ and substrates (CS and/or SBH) are mainly regulated by XYR1 (Table 1).

In CS at $24 \mathrm{~h}$ (where the PBD CAZyme gene expression peaked) in both strains, mainly cellulolytic and xylanolytic genes, but also a few pectinolytic genes were more induced, while additionally in $\Delta x k i 1$ a few xyloglucanolytic genes were higher expressed (Fig. 9a, b). Many of these genes are described as XYR1 target genes $[9,22,27,30]$, including the major cellulolytic genes (e.g., bgl1, egl1/2/3/4/5 and cbh1/2), xylanolytic genes (e.g., xyn $3 / 4$, axe 1 and the candidate $\beta$-xylosidase $/ \alpha-\mathrm{L}-$ arabinofuranosidase Trire2_3739), one pectinolytic gene (pgx1) and one mannanolytic gene (man1) (Table 1). Only in the $\Delta x k i 1$ strain, two arabinanolytic (e.g., abf2) gene and one PCP gene (lxr3) were higher expressed, which were also reported to be XYR1 regulated [9, 22, 30] (Table 1). Expression of $x y r 1$ was also higher, while the cellulase repressor rce1 [31] was lower expressed (Fig. 6). 

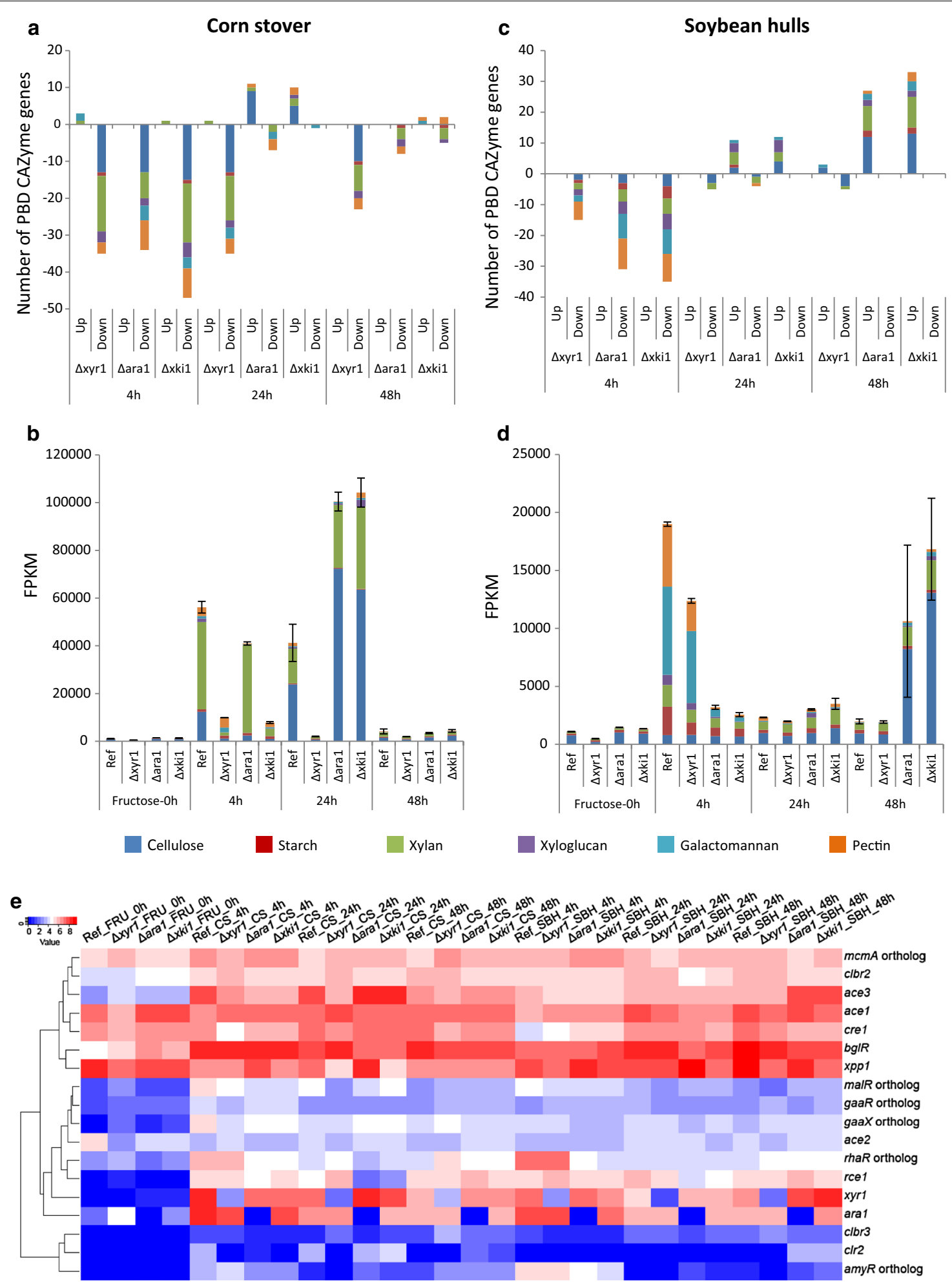

Fig. 9 PBD CAZyme gene expression and TFs hierarchical clustering in the deletion mutants $\triangle x y r 1, \triangle a r a 1$ and $\triangle x k i 1$ compared to the reference strain in corn stover and soybean hulls. a, c Fold change analysis. Differentially expressed genes were those with a $p$ value $\leq 0.05$, fold change $>2.5$ (log2foldchange $>1.32$ ) compared to pre-culture and FPKM $\geq 18$ in at least one condition. $\mathbf{b}, \mathbf{d}$ Total expression analysis were performed with average FPKM level between 3 replicates in plant biomass and 2 replicates in pre-culture. Error bars represent the standard error on the total PBD CAZyme expression. $\mathrm{E}=$ TFs hierarchical clustering 

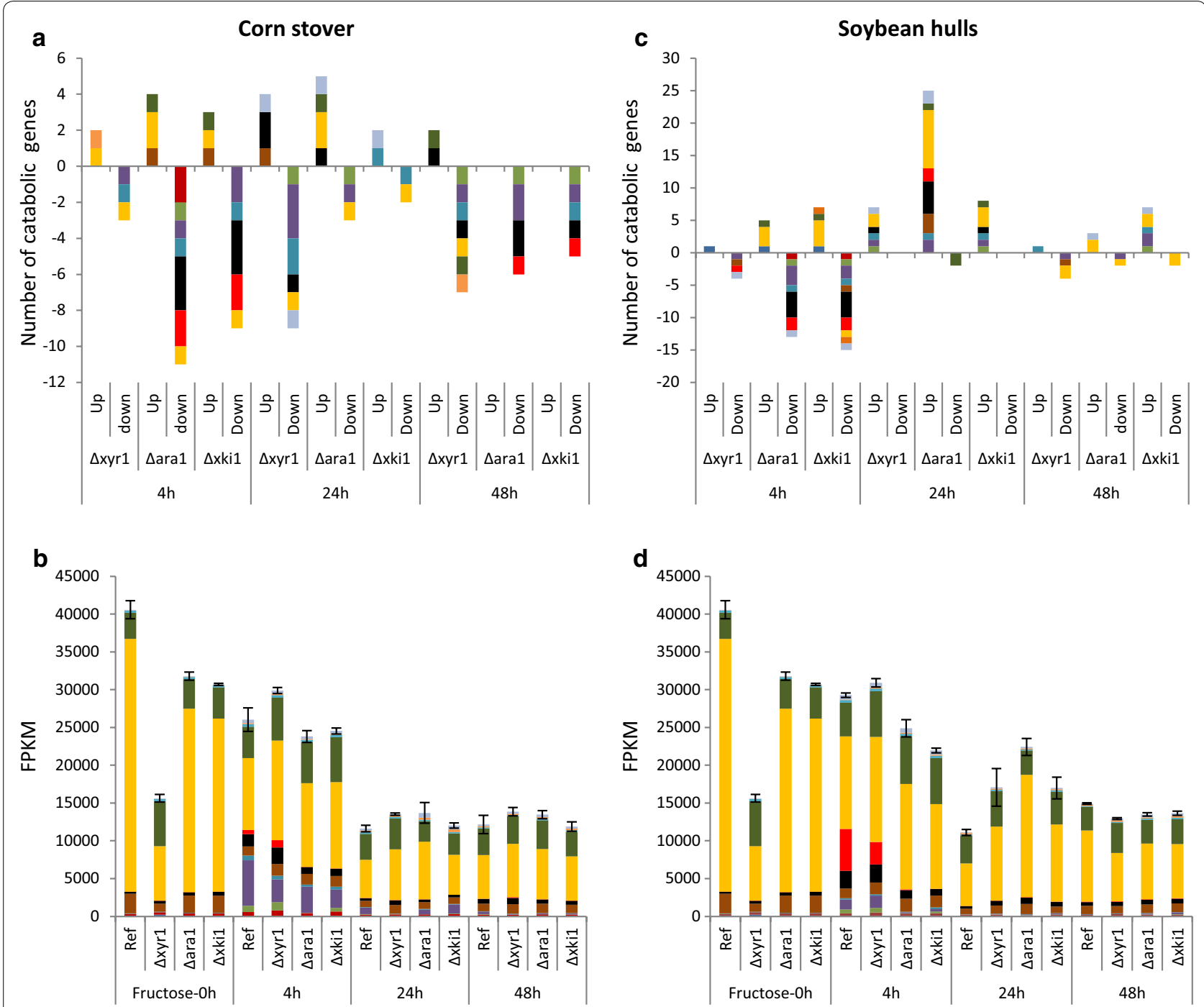

galactofuranose biosynthesis

D-galactose-Leloir pathway

D-galactose-oxido reductive pathway

PCP/D-galactose-oxido reductive pathway $\square$ PCP PPP D-galacturonic acid pathway

L-rhamnose pathway

glycolysis TCA D-mannose pathway D-glucuronic acid pathway alternative L-arabinose pathway

Fig. 10 Carbon catabolic gene expression in the deletion mutants $\Delta x y r 1, \Delta a r a 1$ and $\Delta x k i 1$ compared to the reference strain in corn stover and soybean hulls. a, c Fold change analysis. Differentially expressed genes were those with a p value $\leq 0.05$, fold change $>2.5$ (log2foldchange $>1.32$ ) compared to pre-culture and FPKM $\geq 18$ in at least one condition. $\mathbf{b}, \mathbf{d}$ Total expression analysis were performed with average FPKM level between 3 replicates in plant biomass and 2 replicates in pre-culture. Error bars represent the standard error on the total C-catabolism expression

In $\mathrm{SBH}$ at $48 \mathrm{~h}, \Delta x k i 1$ and $\Delta a r a 1$ had their highest PBD CAZyme gene expression, which included pectinolytic, amylolytic, cellulolytic and xylanolytic genes (Fig. 9). Similar to CS, xyr1 and ace3 were also higher expressed in $\Delta x k i 1$ and $\Delta a r a 1$ (Fig. 6), and many of the genes upregulated in SBH at $48 \mathrm{~h}$ in $\Delta x k i 1$ and $\Delta a r a 1$ have been described as XYR1 target genes $[9,22,27,30]$ (Table 1). These included cellulolytic (e.g., egl1/2/3/4/5 and $c b h 1 / 2$ ), xylanolytic (e.g., $x y n 3 / 45, b x l 1$, axe1 and aes1), and mannanolytic (e.g., man1 and the candidate $\beta$-mannosidase Trire-2_62166) genes $[8,9,18,22,27$, 30] (Table 1). In addition to these, $a b f 1$, the candidate $\beta$-xylosidase $/ \alpha$-L-arabinofuranosidase Trire2_3739, bgl1 (Table 1) and two PCP genes, xyl1 (XYR1 regulated) and $l x r 3$ were higher expressed in $\Delta x k i 1$, but not Dara1 [9]. 
Table 1 Support for xyr1-activation of the PBD CAZymes that were higher expressed in the $\Delta x k i 1$ mutant at later timepoints in corn stover (CS_24 h) and soybean hulls (SBH_48 h)

\begin{tabular}{|c|c|c|c|c|c|}
\hline Trire2_genelD & Gene name & Activity & Regulated by & CS_24h & SBH_48 h \\
\hline 3739 & & $\mathrm{BXL} / \mathrm{ABF}$ & XYR1 & Yes & Yes \\
\hline 22197 & cel1b/bg/1b & $B G L$ & XYR1 & Yes & Yes \\
\hline 46816 & cel3d/bg/3d & $B G L$ & Unknown & Yes & No \\
\hline 49081 & cel74a & XEG & ARA1/XYR1 & Yes & Yes \\
\hline 49976 & egl5/cel45a & EGL & $\mathrm{XYR1}$ & Yes & Yes \\
\hline 56996 & man1 & MAN & XYR1 & No & Yes \\
\hline 60489 & & CUT & Unknown & No & Yes \\
\hline 62166 & & MND & Unknown & No & Yes \\
\hline 69944 & & $\mathrm{AXL} / \mathrm{AGD}$ & ARA1 (XYR1) & No & Yes \\
\hline 72526 & glri/agur & AGU & XYR1 & No & Yes \\
\hline 72567 & cbh2/cel6a & $\mathrm{CBH}$ & XYR1 & & Yes \\
\hline 73632 & axe1 & AXE & XYR1 & No & Yes \\
\hline 73643 & egl4/cel61a & LPMO & XYR1 & Yes & Yes \\
\hline 76210 & $a b f 2$ & $\mathrm{ABF}$ & ARA1(XYR1) & Yes & No \\
\hline 76227 & cel3e/bglise & $B G L$ & Unknown & No & Yes \\
\hline 76672 & bgl1/cel3a/bgl3a & $B G L$ & XYR1 & No & Yes \\
\hline 82227 & $\mathrm{cel} / 3 \mathrm{c} / \mathrm{bg} / 3 \mathrm{c}$ & $B G L$ & XYR1 & No & Yes \\
\hline 82235 & & $A G D$ & XYR1 & No & Yes \\
\hline 103049 & & PGA & ARA1(XYR1) & Yes & No \\
\hline 108477 & & AGD & Unknown & No & Yes \\
\hline 111849 & xyn4 & $X L N$ & XYR1 & No & Yes \\
\hline 112140 & $p g \times 1$ & $P G X$ & Unknown & No & Yes \\
\hline 120229 & xyn3/xyn10a & $X L N$ & XYR1 & No & Yes \\
\hline 120312 & egl2/cel5a & $\mathrm{EGL}$ & XYR1 & No & Yes \\
\hline 120961 & cel61b & LPMO & XYR1 & No & Yes \\
\hline 121127 & $b x / 1 / x y / 3 a$ & $B X L$ & XYR1 & No & Yes \\
\hline 121418 & aes 1 & AES & XYR1 & No & Yes \\
\hline 121735 & cel3b/bg/3b & $B G L$ & XYR1 & No & Yes \\
\hline 122081 & egl1/cel7b & $E G L$ & XYR1 & No & Yes \\
\hline 122780 & $\operatorname{rg} \times 1$ & $R G X$ & ARA1 & No & Yes \\
\hline 123232 & egl3/cel12a & $\mathrm{EGL}$ & XYR1 & No & Yes \\
\hline 123283 & $a b f 1$ & $\mathrm{ABF}$ & ARA1(XYR1) & No & Yes \\
\hline 123818 & xyn2/xyn11a & $X L N$ & XYR1 & No & Yes \\
\hline 123940 & cip2 & GE & XYR1 & No & Yes \\
\hline 123989 & cbh1/cel7a & $\mathrm{CBH}$ & XYR1 & Yes & Yes \\
\hline 124016 & $a g / 2$ & $A G L$ & ARA1 & No & Yes \\
\hline
\end{tabular}

DE genes were assigned to be regulated by ARA1 and/or XYR1 according to literature $[9,22,27,30]$ or this study

ARA1/XYR1, both ARA1 and XYR1 have similar impact in regulation; ARA1 (XYR1), ARA1 has a bigger impact in regulation compared to XYR1

\section{Discussion}

In this study we evaluated the transcriptomic response of the $T$. reesei reference strain, two regulatory mutants and a metabolic mutant during growth on two plant biomass substrates, corn stover (CS, monocot) and soybean hulls (SBH, dicot). Our data showed that substrate composition strongly affected PBD CAZyme expression, resulting in higher induction of a broader set of CAZyme encoding genes in CS compared to SBH (Figs. 3, 4). This higher (hemi-)cellulolytic gene expression matches with the substrate composition (CS is richer in hemicellulose, especially arabinoxylan) (Additional file 1), as well as the more complete set of CAZymes in the $T$. reesei genome for CS degradation [6]. In addition, PBD CAZyme and carbon catabolism related gene expression decreased over time earlier in SBH (Figs. 4, 5), suggesting that release of inducing compounds by $T$. reesei finished earlier on $\mathrm{SBH}$. Indeed, T. reesei has only a few enzyme activities 
[6] for efficient degradation of $\mathrm{SBH}$, such as low numbers of pectinases and $\alpha$-xylosidases and a complete lack of endo-arabinanases and feruloyl esterases. This may have resulted in fewer inducers released from SBH and consequently in lower induction of a narrower set of PBD CAZyme encoding genes. D-xylose, which has been demonstrated to be a major inducer of the (hemi-)cellulolytic system in $T$. reese $i$ [25], is mainly $\alpha$-linked in xyloglucan in SBH, whereas it is $\beta$-linked in xylan in CS [32]. In CS, the major $\beta$-xylosidase $b x l 1$ was highly expressed at all three time points, while no $\beta$-xylosidase expression was found at any time point on SBH (Additional file 4). T. reese $i$ has one putative $\alpha$-xylosidase [6], which was expressed after $4 \mathrm{~h}$ (but not $24 \mathrm{~h}$ and $48 \mathrm{~h}$ ) on SBH, possibly limiting the release of the $\alpha$-linked D-xylose from SBH more so than the $\beta$-linked D-xylose in CS (Additional file 4). This was supported by a study, in which the addition of extra $\alpha$-xylosidase activity to a $T$. reesei commercial enzyme cocktail improved significantly the amount of D-xylose released from pea or tamarind xyloglucan [33].

The data of this study provides leads to improve the production of a commercial $T$. reesei enzyme cocktail, such as by the choice of substrate on which the enzymes are produced and the time after which enzymes are harvest. CS appears a better substrate to obtain a (hemi-)cellulolytic cocktail, while SBH is better for a pectinolytic, amylolytic and mannanolytic cocktail (Fig. 4). In both substrates, the overall expression level was higher at the earliest time point $(4 \mathrm{~h})$, suggesting that high enzyme production will not be sustained over time unless the consumption of potential inducers can be impaired such as in the $\Delta x k i 1$ mutant. A similar pattern was observed in Podospora anserina using comparable methodology including the same substrates [34], where CS induced predominantly (hemi-)cellulases, while $\mathrm{SBH}$ induced more amylolytic and pectinolytic genes. However, in $P$. anserina, SBH was the broader and higher PBD CAZyme gene inducing substrate compared to CS. This indicates that the inducing effect of crude substrates can be species-specific, depending on the genome content and plant biomass degradation strategy. Nevertheless, commonalities can be found in the responses of fungi from diverse biotypes. Analysis of the T. reesei PBD CAZyme genes expressed in both substrates resulted in a core set of 35 shared enzyme activities (data not shown). Of these activities, 14 were in common with the 18 activities in the core set of $P$. anserina and saprobic basidiomycetes $[34,35]$. These 14 activities included three cellulolytic (LPMO, CBH and EGL), one amylolytic (AGD), four xylanolytic (AXE, XLN, ABF, BXL), three mannanolytic (MAN, MND and LAC), two pectinolytic (LAC and $\mathrm{ABF}$ ) and one xyloglucanolytic (XEG) gene. This core enzyme set could be considered as a general response of fungi from diverse biotypes to commonly found components of plant biomass.

We showed that particularly (hemi-)cellulolytic genes were more induced in CS, most likely due to the higher expression of the two (hemi-)cellulolytic regulators $x y r 1$ and ace3 (Fig. 6). However, a higher expression of a TF does not necessarily lead to higher expression of its target genes, because they can be regulated also at posttranscriptional level, as has previously been shown for XYR1 and other TFs [8]. However, our data demonstrates low expression for $c l r 2$ in all conditions, suggesting a different function in T. reesei for CLR2 compared to Neurospora crassa and several Aspergilli, where it has been described as a cellulolytic and mannanolytic regulator [36-38]. Overexpression of $c l r 2$ in T. reesei did not result in substantial enhancement in cellulase and xylanase activity [26]. The orthologs of the $A$. niger D-galacturonic acid regulators gaaR and gaaX [39] were also poorly expressed in most of the conditions (Fig. 6), suggesting that the mechanism with which $T$. reesei responds to D-galacturonic acid may differ from Botrytis cinerea and A. niger $[6,8]$.

Our data showed that all mutations, both regulatory and catabolic, severely affected the initial response $(4 \mathrm{~h})$ to both substrates (Figs. 9, 10). This suggests an initial delay in the release and utilization of enough easily metabolized sugars/inducers at $4 \mathrm{~h}$ by the mutants, resulting in a lack of energy, lower co-factor regeneration (especially for $\Delta x k i 1$ ), such as NADH and NADPH that are necessary for the activity of many oxidoreductases of catabolic pathways, and carbon to synthesize the necessary proteins to degrade plant biomass. Lower PBD CAZyme gene expression was also observed in a study performed with comparable methodology in $A$. niger, where $\Delta x \ln R$ and $\triangle x k i A$ mutants severely affected PBD expression in both CS and SBH at the early stage [40]. Comparison with this $A$. niger study is particularly informative as similar conditions (with respect to timepoints and substrates) and mutants were used.

Our study demonstrates that xyr1 is the major TF affecting CS utilization, where its deletion caused a massive reduction of PBD CAZyme gene expression at $4 \mathrm{~h}$ and $24 \mathrm{~h}$, especially of cellulolytic and xylanolytic genes (Fig. 9). This matches with the substrate composition (Additional file 1) and the function described for this regulator, as indeed CS is richer in (hemi-)cellulose and XYR1 is the main (hemi-)cellulolytic activator [9, 22, 27]. This confirms a previous study, in which another (hemi-) cellulose-rich substrate (wheat bran) and a T. reesei RutC30 Axyr1 mutant were used [18].

In contrast, at $4 \mathrm{~h}$ in $\mathrm{SBH}$, substrate utilization was more affected by the ara 1 deletion resulting in a more severe reduction of PBD CAZyme expression. This dependence 
of the early response to $\mathrm{SBH}$ on ARA1 is most likely due to the fact that ARA1 responds to $\mathrm{L}$-arabinose and D-galactose [9] (inducers which $\mathrm{SBH}$ is richer in Additional file 1 ). During the later response ( $24 \mathrm{~h}$ and $48 \mathrm{~h}$ ), other TFs indirectly compensated for this loss, such as the higher expression of the (hemi-)cellulolytic activators xyr 1 and ace $3[8,22,26]$, or the lower expression of the cellulase repressor rce1 [31] (Fig. 6). Supporting this role of these regulators, cellulolytic and xylanolytic genes were higher expressed in $\Delta a r a 1$ at the later time points (Fig. 9).

Our study is the first which highlights the importance of TFs other than XYR1, in a pectin- and mannan rich substrate such as SBH for T. reesei. Most of the previous studies where $x y r 1$ was deleted used substrates that did not contain pectin or mannan, such as lactose, cellulose or xylan-rich crude plant biomass $[15,18,22,27$, $30,41-44]$. The use of SBH in our study not only showed that XYR1 was not the major regulator on this substrate, but also that XYR1 partially regulates a few pectinolytic and mannanolytic genes (Additional file 4). This suggests that the function of XYR1 is not limited to cellulolytic and xylanolytic genes [27], but is broader than what has so far been described $[8,9,27,30]$. Another broader role for XYR1 was reported by Ma et al. [18], where XYR1 appeared to regulate not only (hemi-)cellulolytic genes, but also genes encoding non-enzymatic cellulose active enzymes, sugar transporters and heat shock proteins.

In A. niger, XlnR (xyr1 ortholog) and AraR (functional homolog of ara1) can compensate for each other's loss, by inducing the target genes of the deleted regulator, most likely due to a similar binding motif [45]. In contrast, $T$. reesei XYR1 and ARA1 are not able to compensate for each other loss, probably because they are not closely related and, therefore, also bind to distinct promoter sequences [8, 9]. XYR1 appeared to express its own target genes, mainly (hemi-)cellulolytic genes [9, 22, 27], to a higher level in the $\triangle$ ara1 strain, but not ARA1-target genes (Fig. 9).

The residual growth of $\Delta x k i 1$ on $\mathrm{L}$-arabinose (which should result in a block of the PCP, Fig. 2), suggests the presence of another catabolic pathway in $T$. reesei to partially catabolize L-arabinose instead of the PCP. In contrast, the $A$. niger xylulokinase mutant cannot grow on L-arabinose [12], indicating a difference in the organization of these pathways between these two fungi. Blast analysis revealed that the $T$. reesei genome contains orthologs for the non-phosphorylative L-arabinose pathway from the bacterium Azospirillum brasiliense [46] (referred to as "alternative L-arabinose pathway" in our analysis). Whether this putative alternative $\mathrm{L}$-arabinose pathway is responsible for the further catabolism of L-arabinose in $\Delta x k i 1$ strain (Fig. 10) requires additional studies.

In $\triangle x k i 1$, the putative block of the PCP resulted in higher PBD CAZyme expression at later time points on both substrates (24 h in CS and $48 \mathrm{~h}$ in SBH) (Fig. 9). Many of these genes were described as XYR1 regulated $[9,27,30]$ (Table 1) and were also upregulated in $\Delta$ ara 1 . A similar inducing effect was also observed in $A$. niger $\triangle x k i A$ [40], where accumulation of inducers [12], such as xylitol and L-arabitol, resulted in more PBD CAZyme genes that were highly expressed compared to the reference strain at later time points. However, this was limited to genes acting on pectin or with an activity that could be involved in the degradation of several substrates. This inducer(s) accumulation could also be the explanation for our results with $T$. reesei, which resulted in higher expression of the (hemi-)cellulolytic regulator xyr1 (Fig. 6) and its target genes [9, 27, 30]. Xylitol and L-arabitol accumulation was already reported for other T. reesei PCP-knockout strains ( $\Delta x y l 1, \Delta l a d 1$, $\Delta l x r 3)$ [13]. This inducer(s) accumulation (such as by xylitol and L-arabitol) is a possible explanation for the higher expression of PBD CAZymes observed also in the $\Delta$ ara1 strain. However, in this case, where a TF is missing, we cannot exclude the involvement of other regulatory mechanism(s) such as secondary/backup regulatory system(s). It was previously shown in $A$. niger that $\mathrm{X} \ln \mathrm{R}$ and AraR have an antagonistic effect on each other and that deletion of one, increases expression of the target genes of the other $[47,48]$. Considering that growth on solid media, where sugars other than pentoses were available, was comparable to the reference strain (Fig. 2), deletion of $x k i 1$ could be used to improve production of $T$. reesei enzyme cocktails at industrial scale by limiting the catabolism of pentose inducers from a crude plant biomass substrate, potentially sustaining the induction of the enzyme encoding genes longer.

\section{Conclusion}

CS induces a broader and higher expression of PBD CAZyme encoding genes in $T$. reese $i$, while SBH could be used to induce an enzyme cocktail that is richer in pectinolytic and mannanolytic enzymes. XYR1 is the major TF affecting CS utilization, while ARA1 affects more SBH utilization. Blocking the PCP by deleting $x k i 1$ leads to higher expression of PBD CAZymes at later time points in the cultures, which could lead to a novel strategy to improve the enzyme cocktail production at industrial level.

\section{Materials and methods}

\section{Strains, media, and growth conditions}

Trichoderma reesei QM9414 (ATCC 26921) [49] was used as reference strain and compared to CBS 143327

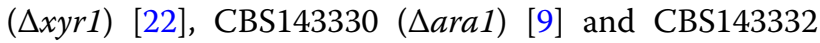
( $\triangle x k i 11)$ (this study) in all experiments. All T. reesei plate cultures were incubated at $28{ }^{\circ} \mathrm{C}$ on PDA (Difco) for sporulation, or minimal medium (MM) [50] with $18 \mathrm{~g} / \mathrm{L}$ 
Select agar (Invitrogen) during the transformation or growth profiling (in this case Na-citrate was removed). The growth profile was performed on MM with $25 \mathrm{mM}$ D-glucose (Sigma), D-fructose (Sigma), D-xylose (Sigma), L-arabinose (Sigma), xylitol (Sigma), L-arabitol (Sigma), D-galactose (Sigma), lactose (Sigma), 1\% arabinan (Megazyme), wheat arabinoxylan (Megazyme), apple pectin (Sigma), avicel (Fluka), 3\% soybean hulls and corn stover in $9 \mathrm{~cm}$ Petri dishes. Duplicate plates were inoculated with $2 \mu \mathrm{L}$ containing $1 \times 10^{3}$ spores, which were pre-germinated overnight in MM with $1 \% \mathrm{D}$-fructose and $0.1 \%$ peptone, and incubated in the dark for at least 5 days at $28{ }^{\circ} \mathrm{C}$. Pre-germination facilitates replicable growth on C-sources where $T$. reesei spores germinate infrequently or do not germinate. Independent deletion strains were generated and tested for growth on a subset of C-sources to confirm the reliability of attributing the observed phenotypes to deleted gene (data not shown). We selected one strain to use in further studies and deposited these at the Westerdijk Fungal Biodiversity Institute collection, with strain number as indicated above.

A transfer experiment was performed for transcriptomics. $250 \mathrm{~mL}$ of complete medium (CM) [51] containing $2 \% \mathrm{D}$-fructose in $1 \mathrm{~L}$ Erlenmeyer flasks was inoculated with $2.5 \times 10^{8}$ fresh spores, harvested from a PDA plate, and incubated in a rotatory shaker at $28{ }^{\circ} \mathrm{C}$ for $20 \mathrm{~h}$ at $250 \mathrm{rpm}$. The mycelium was harvested by filtration, washed with liquid Mandels Andreotti medium (MA) [52] (without carbon source) and 2.5 g mycelium (wet weight) was transferred to $250 \mathrm{~mL}$ Erlenmeyer flasks containing $50 \mathrm{~mL}$ MA with $1 \%$ of soybean hulls or corn stover, and incubated in a rotatory shaker at $28{ }^{\circ} \mathrm{C}$ and $250 \mathrm{rpm}$. After pre-culturing and after $4 \mathrm{~h}, 24 \mathrm{~h}$, and $48 \mathrm{~h}$ of incubation in $\mathrm{CS}$ or SBH, the mycelium was harvested by vacuum filtration, dried between tissue paper, directly frozen in liquid nitrogen and stored at $-45^{\circ} \mathrm{C}$ [53]. All experiments were performed in triplicates, with the exception of precultures, which were performed in duplicates.

\section{Molecular biology methods}

The hygromycin $\mathrm{B}^{\mathrm{R}}$ cassette was amplified from the plasmid pLH1hph [54] and fused with $1 \mathrm{~kb}$ flanking regions up- and downstream of the $x k i 1$ gene by fusion-PCR and purified as described by Klaubauf et al. [53]. This $x k i 1$ deletion cassette was used to transform spores of T. reesei QM9414 $\Delta t k u 70$ [55] by electroporation as described by Schuster et al. [56], using a Bio-Rad Gene Pulser Electroporator System set at $1.8 \mathrm{kV}, 800 \Omega$ and 25 $\mu \mathrm{F}$. DNA from transformants was screened by PCR for the absence of $x k i 1$ and the correct positioning of the insert as described by Klaubauf et al. [53]. The absence of ectopic integrations was confirmed by Southern blot (Additional file 2) using DIG Easy Hyb kit (Roche) and
Anti-Digoxigenin-AP, Fab fragments (Roche) with a probe designed to hybridize to part of the hygromycin resistance gene sequence and amplified with the PCR DIG Probe Synthesis Kit (Roche), according to the manufacturer protocols. Primers used for PCR reactions are listed in Additional file 3.

Total RNA was extracted from mycelium ground in a Tissue Lyser (QIAGEN) using TRIzol reagent (Invitrogen) according to the manufacturer's instructions. RNA integrity and quantity were analyzed on a $1 \%$ agarose electrophoresis gel and with the RNA6000 Nano Assay, using the Agilent 2100 Bioanalyzer (Agilent Technologies) [53].

Culture filtrate samples $(10 \mathrm{~mL})$ were taken after $24 \mathrm{~h}$ and $48 \mathrm{~h}$ and centrifuged for $10 \mathrm{~min}$, at $\sim 10,000 \times g, 4{ }^{\circ} \mathrm{C}$ to separate the solid fraction from the supernatant and stored at $-20{ }^{\circ} \mathrm{C}$. $150 \mu \mathrm{L}$ of these culture filtrates from reference and $\Delta x y r 1$ strains at $24 \mathrm{~h}$ and $48 \mathrm{~h}$ of cultivation were added to $50 \mu \mathrm{L}$ of loading buffer $(10 \%$ of $1 \mathrm{M}$ Tris- $\mathrm{HCl}, \mathrm{pH}$ 6.8; 42\% Glycerol, 4\% (w/v) SDS; $0.02 \%$ (w/v) bromophenol blue; $4 \%$ of $14.7 \mathrm{M}$ Mercaptoethanol), boiled for 2 min to denature the proteins, cooled on ice for $2 \mathrm{~min}$ and centrifuged at $\sim 10,000 \times g$ for $2 \mathrm{~min}$ to remove insoluble material. $20 \mu \mathrm{L}$ was then loaded onto $12 \%(\mathrm{w} / \mathrm{v})$ acrylamide SDS-PAGE gels and a molecular weight marker (Bio-Rad unstained marker) was used to identify the molecular mass of the protein bands. The gels were silver stained [57] and documented using the HP scanner 4400c.

\section{RNA sequencing and read mapping}

RNA samples (5-41 $\mu$ g DNase-treated total RNA) were processed by Joint Genome Institute. RNA sequencing was performed using Illumina HiSeq 2500 (yield $1 \mathrm{~TB}$ of $1 \times 101 \mathrm{bp}$ ). Raw fastq file reads were filtered and trimmed using the JGI QC pipeline. Using BBDuk [BBDuk: https://sourceforge.net/projects/bbmap/] raw reads were evaluated for artifact sequence by kmer matching $(\mathrm{kmer}=25)$, allowing 1 mismatch and detected artifact was trimmed from the $3^{\prime}$ end of the reads. RNA spike-in reads, PhiX reads and reads containing any Ns were removed. Quality trimming was performed using the phred trimming method set at Q6. Reads under the length threshold were removed. Filtered reads from each library were aligned to the reference genome (https:// genome.jgi.doe.gov/Trire2/Trire2.home.html) using HISAT version 0.1.4-beta [58]. featureCounts [59] was used to generate the raw gene counts using gff3 annotations. On average $94 \%$ of the reads mapped to the genome. The RNA-seq data have been deposited at the Sequence Read Archive at NCBI with individual sample BioProject Accession numbers (PRJNA440083 to PRJNA440152 and PRJNA442529 to PRJNA442538. 


\section{RNA-seq data analysis}

Raw gene counts were used to evaluate the level of correlation between biological replicates using Pearson's correlation matrix (Additional file 5). DESeq 2 (version 1.10.0) [60] was used to determine which genes were differentially expressed (DE) between pairs of conditions. The parameters used to call a gene DE between conditions were adjusted $p$ value $\leq 0.05$, foldchange $>2.5$ ( $\log 2$ foldchange $>1.32$ ) and $F P K M \geq 18$ in at least one condition. Genes with FPKM values $<18$ in every condition were considered lowly expressed and ignored in the analysis.

Transcriptomics analysis focused only on genes encoding PBD CAZyme (plant biomass degrading enzymes), carbon catabolic enzymes and TFs (Additional file 4), using the list we built previously $[9,61]$.

PBD CAZyme (which had FPKM $\geq 18$ in at least one of the conditions for a particular heatmap) or TF (all were clustered without regard to their minimum FPKM value) genes were hierarchically clustered using the heatmap.2 function (with default parameters: Euclidean distance, and complete linkage clustering method) from the gplots_3.0.1 package in $\mathrm{R}$ statistical language and environment 3.4.0. Log2 FPKM values were used for the color gradient of the heatmap and FPKM values $<1$ were assigned to 1 .

\section{Additional files}

Additional file 1. Table with sugar composition of corn stover (CS) and soybean hulls (SBH). According to literature lignin content (W/W) is around $15-21 \%$ in CS [19] and 1-4\% in SBH [20].

Additional file 2. Southern blot of $\Delta x k i 1$ strains. Positive gene deletion required bands of $6.4 \mathrm{~kb}$ and $10.8 \mathrm{~kb}$ for $\Delta x \mathrm{ki} 1$. Both $\Delta x \mathrm{ki}$ strains were correct.

Additional file 3. Table with PCR primers used in this study.

Additional file 4. Enzyme activity abbreviations and transcriptome dataset tables. Table with enzyme activity, their abbreviations and their predicted target substrate used in this study. The same color scheme for target substrates was used in the main text. A gene to be assigned as expressed more on a substrate ( $\mathrm{CS}$ or $\mathrm{SBH}$ ) requires a foldchange $>2.5$, $p \leq 0.05$ and at least 18 FPKM of expression in at least one condition. Genes with expression < 18 FPKM in each condition were excluded from subsequent analysis, because they were considered to be too poorly expressed.

Additional file 5. Pearson correlation matrix of Trichoderma reesel transcriptomes. Raw gene counts were used to evaluate the level of correlation between biological replicates using Pearson's correlation. Pearson correlation matrix were performed in R (v3.4.0) statistical language and environment, the core function from the stats base package and the corrplot (v 0.77) package were used for the analysis. One sample (reference strain on $\mathrm{SBH}$ at $48 \mathrm{~h}$ ) was removed from the dataset because it correlated poorly with its replicates.

Additional file 6 . Analysis of the protein banding patterns from the three replicate culture supernatants of $T$. reesei reference (ref) and $\Delta x y r 1$ strains cultured with either corn stover or soybean hulls for $24 \mathrm{~h}$ and $48 \mathrm{~h}$. The same volume of culture supernatant was loaded for all samples.

\section{Abbreviations}

TF: transcription factor; CAZy: carbohydrate-active enzymes database; CAZyme: carbohydrate-active enzyme; CCR: carbon catabolite repression; PBD: plant biomass degrading enzyme; CS: corn stover; SBH: soybean hulls; PCP: pentose catabolic pathway; PPP: pentose phosphate pathway; ABF: a-L-arabinofuranosidase; AES: acetyl esterase; AGD: a-1,4-glucosidase; AXE: acetyl xylan esterase; BXL: $\beta$-1,4-xylosidase; CBH: cellobiohydrolase; EGL: $\beta$-1,4-endoglucanase; LAC: $\beta$-1,4-galactosidase; LPMO: lytic polysaccharide monooxygenase; MAN: $\beta$-1,4-endomannanase; MND: $\beta$-1,4-mannosidase; XEG: xyloglucan-active endo- $\beta$-1,4-glucanase.

\section{Authors' contributions}

TB and RJML carried out the experiments. TB analyzed the data and wrote the manuscript. MVAP performed part of the bioinformatics analysis. RSK generated the $x$ ki1 deletion strains. RPdV designed the study. RPdV and PD supervised the experiments and the manuscript. BS reviewed the manuscript and provided T. reesei QM9414 strains ATCC 26921 (reference), $\Delta t k u 70$ and $\triangle x y r 1 . \mathrm{KL}, \mathrm{MW}, \mathrm{AL}, \mathrm{VN}$ and IVG performed RNA sequencing. All authors read and approved the final manuscript.

\section{Author details}

${ }^{1}$ Fungal Physiology, Westerdijk Fungal Biodiversity Institute \& Fungal Molecular Physiology, Utrecht University, Uppsalalaan 8, 3584 CT Utrecht, The Netherlands. ${ }^{2}$ US Department of Energy Joint Genome Institute, 2800 Mitchell Drive, Walnut Creek, CA 94598, USA. ${ }^{3}$ Department of Plant and Microbial Biology, University of California Berkeley, Berkeley, CA 94598, USA. ${ }^{4}$ Research Area Biochemical Technology, Institute of Chemical, Environmental and Bioscience Engineering, TU Wien, 1060 Vienna, Austria.

\section{Acknowledgements}

Not applicable.

\section{Competing interests}

The authors declare that they have no competing interests.

\section{Availability of data and materials}

The RNA-seq data have been deposited at the Sequence Read Archive at NCBI with individual sample BioProject Accession Numbers PRJNA440083 to PRJNA440152 and PRJNA442529 to PRJNA442538.

\section{Consent for publication}

Not applicable.

\section{Ethics approval and consent to participate} Not applicable.

\section{Funding}

This study was supported by a Grant of the Applied Science Division of NWO, and the Technology Program of the Ministry of Economic Affairs 016.130.609 to RPdV. PD was supported by a Grant from the ALW division of NWO (824.15.023) to RPdV. BS was supported by the Austrian Science Fund project P24219. The work conducted by the U.S. Department of Energy Joint Genome Institute $(J \mathrm{Gl})$, was supported by the Office of Science of the U.S. Department of Energy under Contract No. DE-AC02-05CH11231.

\section{Publisher's Note}

Springer Nature remains neutral with regard to jurisdictional claims in published maps and institutional affiliations.

Received: 20 December 2018 Accepted: 3 April 2019

Published online: 09 April 2019

\section{References}

1. Makela MR, Donofrio N, de Vries RP. Plant biomass degradation by fungi. Fungal Genet Biol. 2014;72:2-9. 
2. Bischof RH, Ramoni J, Seiboth B. Cellulases and beyond: the first 70 years of the enzyme producer Trichoderma reesei. Microb Cell Fact. 2016;15(1):106.

3. Druzhinina IS, Kubicek CP. Genetic engineering of Trichoderma reesei cellulases and their production. Microb Biotechnol. 2017;10(6):1485-99.

4. Peterson R, Nevalainen H. Trichoderma reesei RUT-C30-thirty years of strain improvement. Microbiology. 2012;158(Pt 1):58-68.

5. Kubicek CP. Systems biological approaches towards understanding celIulase production by Trichoderma reesei. J Biotechnol. 2013;163(2):133-42.

6. Martinez D, Berka RM, Henrissat B, Saloheimo M, Arvas M, Baker SE, Chapman J, Chertkov O, Coutinho PM, Cullen D, et al. Genome sequencing and analysis of the biomass-degrading fungus Trichoderma reesei (syn. Hypocrea jecorina). Nat Biotechnol. 2008;26(5):553-60.

7. Gupta VK, Steindorff AS, de Paula RG, Silva-Rocha R, Mach-Aigner AR, Mach RL, Silva RN. The post-genomic Era of Trichoderma reesei: what's Next? Trends Biotechnol. 2016;34(12):970-82.

8. Benocci T, Aguilar-Pontes MV, Zhou M, Seiboth B, de Vries RP. Regulators of plant biomass degradation in ascomycetous fungi. Biotechnol Biofuels. 2017; 10:152.

9. Benocci T, Aguilar-Pontes MV, Kun RS, Seiboth B, de Vries RP, Daly P. ARA1 regulates not only L-arabinose but also D-galactose catabolism in Trichoderma reesei. FEBS Lett. 2018;592(1):60-70.

10. Seiboth B, Metz B. Fungal arabinan and L-arabinose metabolism. Appl Microbiol Biotechnol. 2011;89(6):1665-73.

11. Khosravi C, Benocci T, Battaglia E, Benoit I, de Vries RP. Sugar catabolism in Aspergillus and other fungi related to the utilization of plant biomass. Adv Appl Microbiol. 2015;90:1-28.

12. Witteveen CFB, Busink R, Vondervoort PVD, Dijkema C, Swart K, Visser J. L-arabinose and D-xylose catabolism in Aspergillus niger. J Gen Microbiol. 1989;135:2163-71.

13. Herold S, Bischof R, Metz B, Seiboth B, Kubicek CP. Xylanase gene transcription in Trichoderma reesei is triggered by different inducers representing different hemicellulosic pentose polymers. Eukaryot Cell. 2013;12(3):390-8.

14. Hong Y, Dashtban M, Kepka G, Chen S, Qin W. Overexpression of D-xylose reductase $(x y / 1)$ gene and antisense inhibition of $D-x y l u l o k i n a s e ~(x y i H)$ gene increase xylitol production in Trichoderma reesei. Biomed Res Int. 2014:2014:169705.

15. Bischof R, Fourtis L, Limbeck A, Gamauf C, Seiboth B, Kubicek CP. Comparative analysis of the Trichoderma reesei transcriptome during growth on the cellulase inducing substrates wheat straw and lactose. Biotechnol Biofuels. 2013;6(1):127.

16. Borin GP, Sanchez CC, de Santana ES, Zanini GK, Dos Santos RAC, de Oliveira Pontes A, de Souza AT, Dal'Mas R, Riano-Pachon DM, Goldman $\mathrm{GH}$, et al. Comparative transcriptome analysis reveals different strategies for degradation of steam-exploded sugarcane bagasse by Aspergillus niger and Trichoderma reesei. BMC Genomics. 2017;18(1):501.

17. Ries L, Pullan ST, Delmas S, Malla S, Blythe MJ, Archer DB. Genome-wide transcriptional response of Trichoderma reesei to lignocellulose using RNA sequencing and comparison with. BMC Genomics. 2013;14:541.

18. Ma L, Chen L, Zhang L, Zou G, Liu R, Jiang Y, Zhou Z. RNA sequencing reveals $X y r 1$ as a transcription factor regulating gene expression beyond carbohydrate metabolism. Biomed Res Int. 2016;2016:4841756.

19. Buranov AU, Mazza G. Lignin in straw of herbaceous crops. Ind Crop Prod. 2008;28(3):237-59.

20. Mielenz JR, Bardsley JS, Wyman CE. Fermentation of soybean hulls to ethanol while preserving protein value. Bioresour Technol. 2009;100(14):3532-9.

21. Akel E, Metz B, Seiboth B, Kubicek CP. Molecular regulation of arabinan and L-arabinose metabolism in Hypocrea jecorina (Trichoderma reesei). Eukaryot Cell. 2009;8(12):1837-44

22. Stricker AR, Grosstessner-Hain K, Wurleitner E, Mach RL. Xyr1 (xylanase regulator 1) regulates both the hydrolytic enzyme system and D-xylose metabolism in Hypocrea jecorina. Eukaryot Cell. 2006;5(12):2128-37.

23. Hasper AA, Visser J, de Graaff LH. The Aspergillus niger transcriptional activator $X \ln R$, which is involved in the degradation of the polysaccharides xylan and cellulose, also regulates D-xylose reductase gene expression. Mol Microbiol. 2000;36:193-200.

24. Mach-Aigner AR, Omony J, Jovanovic B, van Boxtel AJ, de Graaff LH. D-xylose concentration-dependent hydrolase expression profiles and the function of CreA and XInR in Aspergillus niger. Appl Environ Microbiol. 2012;78(9):3145-55.

25. Mach-Aigner AR, Pucher ME, Mach RL. D-xylose as a repressor or inducer of xylanase expression in Hypocrea jecorina (Trichoderma reesei). Appl Environ Microbiol. 2010;76(6):1770-6.

26. Hakkinen M, Valkonen MJ, Westerholm-Parvinen A, Aro N, Arvas M, Vitikainen M, Penttila M, Saloheimo M, Pakula TM. Screening of candidate regulators for cellulase and hemicellulase production in Trichoderma reesei and identification of a factor essential for cellulase production. Biotechnol Biofuels. 2014;7(1):14.

27. Mach-Aigner AR, Pucher ME, Steiger MG, Bauer GE, Preis SJ, Mach RL. Transcriptional regulation of $x y r 1$, encoding the main regulator of the xylanolytic and cellulolytic enzyme system in Hypocrea jecorina. Appl Environ Microbiol. 2008;74(21):6554-62.

28. Gruben BS, Zhou M, Wiebenga A, Ballering J, Overkamp KM, Punt PJ, de Vries RP. Aspergillus niger RhaR, a regulator involved in L-rhamnose release and catabolism. Appl Microbiol Biotechnol. 2014;98(12):5531-40.

29. Derntl C, Kluger B, Bueschl C, Schuhmacher R, Mach RL, Mach-Aigner AR. Transcription factor Xpp1 is a switch between primary and secondary fungal metabolism. Proc Natl Acad Sci USA. 2017;114(4):E560-9.

30. Portnoy T, Margeot A, Seidl-Seiboth V, Le Crom S, Ben Chaabane F, Linke $\mathrm{R}$, Seiboth B, Kubicek CP. Differential regulation of the cellulase transcription factors XYR1, ACE2, and ACE1 in Trichoderma reesei strains producing high and low levels of cellulase. Eukaryot Cell. 2011;10(2):262-71.

31. Cao Y, Zheng F, Wang L, Zhao G, Chen G, Zhang W, Liu W. Rce1, a novel transcriptional repressor, regulates cellulase gene expression by antagonizing the transactivator Xyr1 in Trichoderma reesei. Mol Microbiol. 2017; 105(1):65-83.

32. Andersen MR, Giese M, de Vries RP, Nielsen J. Mapping the polysaccharide degradation potential of Aspergillus niger. BMC Genomics. 2012;13:313.

33. Jabbour D, Borrusch MS, Banerjee G, Walton JD. Enhancement of fermentable sugar yields by alpha-xylosidase supplementation of commercial cellulases. Biotechnol Biofuels. 2013;6(1):58

34. Benocci T, Daly P, Aguilar-Pontes M, Lail K, Wang M, Lipzen A, Vivian N, Grigoriev I, de Vries R. Enzymatic adaptation of Podospora anserina to different plant biomass provides leads to optimized commercial enzyme cocktails. Biotechnol J. 2018 (in press).

35. Peng M, Aguilar-Pontes MV, Hainaut M, Henrissat B, Hilden K, Makela MR, de Vries RP. Comparative analysis of basidiomycete transcriptomes reveals a core set of expressed genes encoding plant biomass degrading enzymes. Fungal Genet Biol. 2017;112:40-6.

36. Coradetti ST, Craig JP, Xiong Y, Shock T, Tian C, Glass NL. Conserved and essential transcription factors for cellulase gene expression in ascomycete fungi. Proc Natl Acad Sci USA. 2012;109(19):7397-402.

37. Raulo R, Kokolski M, Archer DB. The roles of the zinc finger transcription factors XInR, ClrA and CIrB in the breakdown of lignocellulose by Aspergillus niger. AMB Exp. 2016;6(1):5

38. Ogawa M, Kobayashi T, Koyama Y. ManR, a transcriptional regulator of the beta-mannan utilization system, controls the cellulose utilization system in Aspergillus oryzae. Biosci Biotechnol Biochem. 2013;77(2):426-9.

39. Niu J, Alazi E, Reid ID, Arentshorst M, Punt PJ, Visser J, Tsang A, Ram AF. An evolutionarily conserved transcriptional activator-repressor module controls expression of genes for D-galacturonic acid utilization in Aspergillus niger. Genetics. 2017;205(1):169-83.

40. Khosravi C KJ, Battaglia E, Aguilar-Pontes MV, Lipzen A, Ng V, Grigoriev I, de Vries RP: Chapter 4. Transcriptome analysis of Aspergillus niger XInR and $x$ kiA mutants grown on corn stover and soybean hulls. PhD thesis. Utrecht, The Netherlands: Utrecht University; 2017.

41. Mello-de-Sousa TM, Rassinger A, Derntl C, Pocas-Fonseca MJ, Mach $\mathrm{RL}$, Mach-Aigner AR. The relation between promoter chromatin status, Xyr 1 and cellulase expression in Trichoderma reesei. Curr Genomics. 2016;17(2):145-52

42. Lichius A, Seidl-Seiboth V, Seiboth B, Kubicek CP. Nucleo-cytoplasmic shuttling dynamics of the transcriptional regulators XYR1 and CRE1 under conditions of cellulase and xylanase gene expression in Trichoderma reesei. Mol Microbiol. 2014;94(5):1162-78.

43. Stricker AR, Steiger MG, Mach RL. Xyr1 receives the lactose induction signal and regulates lactose metabolism in Hypocrea jecorina. FEBS Lett. 2007;581(21):3915-20.

44. Dos Santos Castro L, de Paula RG, Antonieto AC, Persinoti GF, Silva-Rocha $\mathrm{R}$, Silva RN. Understanding the Role of the Master Regulator XYR1 in 
Trichoderma reesei by Global Transcriptional Analysis. Front Microbiol. 2016;7:175.

45. Battaglia E, Visser L, Nijssen A, van Veluw GJ, Wosten HA, de Vries RP. Analysis of regulation of pentose utilisation in Aspergillus niger reveals evolutionary adaptations in Eurotiales. Stud Mycol. 2011;69(1):31-8.

46. Watanabe S, Kodaki T, Makino K. Cloning, expression, and characterization of bacterial L-arabinose 1-dehydrogenase involved in an alternative pathway of L-arabinose metabolism. J Biol Chem. 2006;281 (5):2612-23.

47. Battaglia E, Visser L, Nijssen A, van Veluw J, Wösten HAB, de Vries RP. Analysis of regulation of pentose utilisation in Aspergillus niger reveals evolutionary adaptations in the Eurotiales. Stud Mycol. 2011:69:31-8.

48. de Groot MJL, van de Vondervoort PJl, de Vries RP, vanKuyk PA, Ruijter GJG, Visser J. Isolation and characterization of two specific regulatory Aspergillus niger mutants shows antagonistic regulation of arabinan and xylan metabolism. Microbiology. 2003;149:1183-91.

49. Montenecourt BS, Eveleigh DE. Preparation of mutants of Trichoderma reesei with enhanced cellulase production. Appl Environ Microbiol. 1977:34(6):777-82.

50. Penttila M, Nevalainen H, Ratto M, Salminen E, Knowles J. A versatile transformation system for the cellulolytic filamentous fungus Trichoderma reesei. Gene. 1987;61 (2):155-64.

51. de Vries RP, Burgers K, van de Vondervoort PJ, Frisvad JC, Samson RA, Visser J. A new black Aspergillus species, A. vadensis, is a promising host for homologous and heterologous protein production. Appl Environ Microbiol. 2004;70(7):3954-9.

52. Mandels M, Andreotti RE. Problems and challenges in the cellulose to cellulase fermentation. Process Biochem. 1978:13:6.
53. Klaubauf S, Zhou M, Lebrun MH, de Vries RP, Battaglia E. A novel L-arabinose-responsive regulator discovered in the rice-blast fungus Pyricularia oryzae (Magnaporthe oryzae). FEBS Lett. 2016;590(4):550-4.

54. Hartl L, Kubicek CP, Seiboth B. Induction of the gal pathway and cellulase genes involves no transcriptional inducer function of the galactokinase in Hypocrea jecorina. J Biol Chem. 2007;282(25):18654-9.

55. Guangtao Z, Hartl L, Schuster A, Polak S, Schmoll M, Wang T, Seidl V, Seiboth B. Gene targeting in a nonhomologous end joining deficient Hypocrea jecorina. J Biotechnol. 2009;139(2):146-51.

56. Schuster A, Bruno KS, Collett JR, Baker SE, Seiboth B, Kubicek CP, Schmoll $M$. A versatile toolkit for high throughput functional genomics with Trichoderma reesei. Biotechnol Biofuels. 2012;5(1):1.

57. Chevallet M, Luche S, Rabilloud T. Silver staining of proteins in polyacrylamide gels. Nat Protoc. 2006;1 (4):1852-8.

58. Kim D, Langmead B, Salzberg SL. HISAT: a fast spliced aligner with low memory requirements. Nat Methods. 2015;12(4):357-60.

59. Liao Y, Smyth GK, Shi W. featureCounts: an efficient general purpose program for assigning sequence reads to genomic features. Bioinformatics. 2014;30(7):923-30.

60. Love MI, Huber W, Anders S. Moderated estimation of fold change and dispersion for RNA-seq data with DESeq2. Genome Biol. 2014;15(12):550.

61. Hakkinen M, Arvas M, Oja M, Aro N, Penttila M, Saloheimo M, Pakula TM. Re-annotation of the CAZy genes of Trichoderma reesei and transcription in the presence of lignocellulosic substrates. Microb Cell Fact. 2012;11:134.
Ready to submit your research? Choose BMC and benefit from:

- fast, convenient online submission

- thorough peer review by experienced researchers in your field

- rapid publication on acceptance

- support for research data, including large and complex data types

- gold Open Access which fosters wider collaboration and increased citations

- maximum visibility for your research: over 100M website views per year

At BMC, research is always in progress.

Learn more biomedcentral.com/submissions 